\title{
Phase Transitions and Critical Fluctuations in the Visual Coordination of Rhythmic Movements Between People
}

\author{
R. C. Schmidt, Claudia Carello, and M. T. Turvey \\ Center for the Ecological Study of Perception and Action, University of Connecticut, Storrs, \\ and Haskins Laboratories, New Haven, Connecticut
}

\begin{abstract}
By watching each other's lower oscillating leg, 2 seated Ss kept a common tempo and a particular phase relation of either $0^{\circ}$ (symmetric mode) or $180^{\circ}$ (alternate mode). This study investigated the differential stability of the 2 phase modes. In Experiment 1, in which Ss were instructed to remain in the initial phase mode, the alternate phase mode was found to be less stable as the frequency of oscillation increased. In addition, analysis of the nonsteady state cycles revealed evidence of a switching to the symmetric phase mode for the initial alternate phase mode trials. In Experiments 2 and 3, Ss were instructed to remain at a noninitial phase angle if it was found to be more comfortable. The transition observed between the 2 phase modes satisfies the criteria of a physical bifurcation - hysteresis, critical fluctuations, and divergence-and is consonant with previous findings on transitions in limb coordination within a person.
\end{abstract}

The coordination of movements between people is an omnipresent aspect of daily life. Such coordinations consist of the very natural and commonplace coordinations exhibited by people walking and talking together and the very practiced and refined coordinations exhibited by people playing sports or music, or dancing. The degree of coordination between basketball players moving downcourt or the degree of coordination between two ballet dancers is quite obvious. The coordination of movements between speaker and listener (Kendon, 1970), however, or between mother and infant (Bernieri, Reznick, \& Rosenthal, 1988) is more subtle and is apparent only through study. In all such cases a coordinative relationship is formed through an interaction of two individuals in order to produce some goal (e.g., score a basket, have a conversation). The unique challenge for an account of between-persons coordination-the durations, spacings, and phasings of movements and their components-resides in the fact that the two individuals share neither a common cognitive nor neural mechanism.

Because of the cooperative nature of the relationship, the two individuals can be thought of as a single organism (Asch, 1952; Newtson, Hairfield, Bloomingdale, \& Cutino, 1987), a dyadic synergy. A common property of dyadic synergies is the entrainment of rhythmicities (Davis, 1982; Newtson et

Two of the experiments reported here were part of a dissertation presented to the University of Connecticut by R. C. Schmidt. The research was supported in part by a University of Connecticut Doctoral Fellowship awarded to R. C. Schmidt, National Institutes of Health Grant BRS-RR-05596 awarded to Haskins Laboratories, and National Science Foundation (NSF) Grant BNS-8811510 awarded to M. T. Turvey. During the preparation of the article, Claudia Carello was supported by NSF Grant BNS-8720144 and M. T. Turvey held a James McKeen Cattell Fellowship.

The authors wish to acknowledge the helpful suggestions of John Scholz and Gregor Schöner at various stages of the research.

Correspondence concerning this article should be addressed to $R$. C. Schmidt, CESPA, Box U-20, 406 Babbidge Road, University of Connecticut, Storrs, Connecticut 06269. al., 1987); that is, the spatial and temporal aspects of the two individuals' movements are related congruently-they are entrained-and the movements repeat (either periodically or stochastically)-they are rhythmic. Entrainment and sustained periodic behavior are properties of nonlinear dissipative systems, and it is from this dynamical perspective that we will attempt to understand between-persons coordination.

The experiments reported in this article are directed at the questions of whether certain entrainment phenomena found in within-person coordination also hold for between-persons coordination, and whether the same very general dynamical principles govern both. There are two kinds of entrainment that two physically coupled oscillators can enter into. When the two oscillators are related stably in their timing, they are said to be frequency entrained. When they are related stably in their spatial positions within a cycle, they are said to be phase entrained or phase locked. A perturbation of the oscillators in the former case will produce a return to the preperturbation frequency, and in the latter case to the preperturbation relative phase angle. Dynamically speaking such behavior reflects the existence of an attractor. Assuming that a set of variables has been found to define the coordinate space (state space) of the dynamical system's behavior, an attractor is a set of points (the limit set) within the state space that the system will settle on if unperturbed for a long period of time. Investigations of bimanual within-person coordination have yielded evidence that this kind of dynamical entity, an attractor, is a basic control structure that underlies frequency entrainment and phase locking found in the coordination of limbs. This study investigates whether such a dynamical interpretation can be given to the visually coordinated phasing of limbs between two people.

The hypothesis that the coordination of limbs is the outcome of nonlinear dissipative dynamics was proposed originally by Kugler and his colleagues (Kelso, Holt, Rubin, \& Kugler, 1981; Kugler, Kelso, \& Turvey, 1980, 1982; Kugler, Turvey, \& Shaw, 1982). It has since been advanced on several fronts, both theoretical and empirical, by a number of investigators (e.g., Beek, 1989; Beek \& Beek, 1988; Haken, Kelso, 
\& Bunz, 1985; Kay, 1986, 1988; Kay, Kelso, Saltzman, \& Schöner, 1987; Kelso, Schöner, Scholz, \& Haken, 1987; Kugler, 1986; Kugler \& Turvey, 1987, 1988; Saltzman, 1987; Schöner \& Kelso, 1988a, 1988c; Turvey, Rosenblum, Schmidt, \& Kugler, 1986). The theoretical claim is that organisms have evolved to balance the extraordinary burden of coordination between their cognitive and/or neural mechanisms and the principles of self-organization found in the dynamical properties of their movement systems as physical systems (Kugler \& Turvey, 1987).

Recognizing that coordinated movements involve many variables of cellular, muscular, and skeletal origin, the dynamical perspective aims at understanding how these biological state spaces of high dimensionality reduce lawfully to control spaces of low dimensionality. Or alternatively, it aims to understand how the many degrees of freedom of the movement system's physiology become compressed so that they can be controlled by an intentional agent. The dynamical entity of an attractor has been suggested as the means by which this necessary compressing of degrees of freedom occurs (Saltzman \& Kelso, 1987). For a dynamical attractor to operate in the coordination of the movements of an organism, the organism must interact with it in two ways. First, the organism must set up the conditions that will allow the dynamical control structure to emerge, such as intentional constraints that allow muscle synergies and their concomitant metabolic engine processes to be formed. Second, the organism must "tune" the control structure through a parameterization of the control structure's functionally relevant properties to accomplish a given task in a changing environment. The interaction between the dynamical control structure and the organism can be interpreted in a number of different ways-neural, cognitive, or perceptual-depending on the perspective of the researcher. The claim that an organism's movement system has a dynamical organization can be accommodated by any of these theoretical positions. The perspective subscribed to here is one based on the Gibsonian concept of information as morphological form (Kugler et al., 1980). It has been suggested that the formation (Kugler \& Turvey, 1987) and tuning (Fitch, Tuller, \& Turvey, 1982) of coordinated states are inherently perceptual processes, based on the perception of dynamic properties of the actor's body segments, dynamic properties of the environment, and the relations between the actor and the environment.

\section{Dynamical Basis of the Phasing of Limbs Within a Person}

The argument for a dynamical basis of the phasing observed in the coordination of oscillating limbs begins with the evidence that only two stable relative phase angles (or phase modes) are observed, one at $0^{\circ}$ relative phase angle (symmetric mode) and one at $180^{\circ}$ relative phase angle (alternate mode; Kelso et al., 1987; Stafford \& Barnswell, 1985; von Holst, 1939/1973). The symmetric phase mode occurs when the limbs are at the same point of their cycles at the same time (Limb 1-Limb 2: flexion-flexion, extension-extension), and the alternate phase mode occurs when the limbs are in op- posite points of their cycles at the same time (Limb 1-Limb 2: flexion-extension, extension-flexion). That only two modes of phase locking are observed suggests that there are perhaps two attractors underlying phase entrainment, whose limit sets are at $0^{\circ}$ and $180^{\circ}$ relative phase.

If a dynamical system is forced through some parameterization away from its limit set, as is often the case in systems open to outside energy sources (Prigogine, 1980; Prigogine \& Stengers, 1984), the behavior of the system becomes less stable. Yaminishi, Kawato, and Suzuki (1980) have noted in bimanual finger-tapping experiments that tapping at metronome-driven phase differences other than $0^{\circ}$ and $180^{\circ}$ produces greater deviations from the intended phase and greater fluctuations. They also found that the fluctuations at the inbetween phase differences decrease in the direction of the phase differences of $0^{\circ}$ and $180^{\circ}$. These results are again consistent with the claim that there are two point attractors underlying the phasing of movements at $0^{\circ}$ and $180^{\circ}$ that draw nearby trajectories toward them. A dynamical model of this attractor landscape has been presented by Kelso et al. (1987) that predicts the observed patternings of deviations from the intended phase and fluctuations found by Yaminishi et al. (1980).

The limbs of the same girdle in locomoting quadrupeds relate in either the alternate mode at low-speed gaits such as the walk or the symmetric mode at high-speed gaits such as the gallop. Sharp transitions occur between one gait and another as the speed of locomotion increases; and these transitions within a girdle correspond to a transition from an alternate mode of phasing to a symmetric mode of phasing. Hoyt and Taylor (1981) have provided evidence that such gait transitions have a dynamical basis: The gait transitions occur in quadrupeds to produce the least energetically costly coordination for a given speed. ${ }^{1}$ For bimanual oscillations of the wrists or index fingers, Kelso and his associates (Kelso, 1984; Kelso \& Scholz, 1985) found that the alternate phase mode becomes unstable before a transition to the symmetric phase mode when the frequency of oscillation is increased. No transition is observed when the frequency is increased starting in the symmetric phase mode. This kind of nonlinear change in phase-locked modes has the characteristic property of a physical bifurcation or catastrophe: a one-way sudden jump between two modes of functioning with inaccessible regions between the two modes (Gilmore, 1981; Haken, 1978). Such a jump is caused by the annihilation of the attractor at the $180^{\circ}$ phase mode as the frequency of oscillation is increased. Kelso and his colleagues presented a model of this phenomenon as a physical bifurcation (Haken et al., 1985; Schöner, Haken, \& Kelso, 1986) and tested the model's various predictions affirmatively (Kelso, Scholz, \& Schöner, 1986; Scholz, Kelso, \& Schöner, 1987).

\footnotetext{
${ }^{1}$ Although humans do not exhibit transitions in the phasing of their limbs as quadrupeds do (for obvious reasons), they exhibit many more transitions in the phase locking of their limb and respiratory rhythms than do quadrupeds. Bramble and Carrier (1983) have suggested that this capacity of humans to change limb/respiratory phase locking is perhaps an alternative strategy for regulating energetic cost.
} 
The nonlinear jump from the $180^{\circ}$ phase mode to the $0^{\circ}$ phase mode that occurs with increasing frequency has the structure of a physical bifurcation, and as such is further evidence for a dynamical control structure governing the phasing of limbs. This phenomenon also demonstrates that the alternate phase mode is intrinsically less stable than the symmetric phase mode. Kelso et al. (1986) have shown that before the bifurcation, the fluctuations in relative phase increase dramatically for the alternate mode but remain constant for the symmetric mode at all frequencies of oscillation. This differential stability of the two modes has been interpreted as a reflection of the differential topology of the two phase attractors (Haken et al., 1985).

In summary, evidence exists to indicate that the phasing of limbs within a person is dynamically organized. The control structure governing phasing has an attractor landscape with limit points at $0^{\circ}$ relative phase and $180^{\circ}$ relative phase. The relative phases in between are catchment regions (Thompson \& Stewart, 1986) for these two point attractors: A trajectory perturbed to any other phase angle between $0^{\circ}$ and $180^{\circ}$ will proceed to one of these limit points. But the stability or attractiveness of the two phase attractors is not equal (Turvey et al., 1986). The alternate mode attractor is less stable, especially as the frequency of oscillation is increased when a complete annihilation of the steady state is observed.

\section{Contrasting Circumstances of Within-Person and Between-Persons Coordination}

This study investigates whether the control structure underlying the phasing of oscillating limbs visually coordinated between two people is dynamical. Is the dynamical solution to the degrees of freedom problem (stated earlier) for withinperson coordination so general that it will apply to the coordination of the movements of two people as well? Some studies that have investigated the cooperative activities of social organisms suggest that this is a possibility. For example, both termite nest building and bird flock organization in flight have been modeled dynamically (Kugler \& Turvey, 1987).

The two cases of between-persons and within-person coordinations differ in many respects. Most notably, they differ in the populations of neurons involved (the nervous systems of two people vs. the nervous system of one person) and in the perceptual system that is primarily involved in achieving the coordination (the visual perceptual system in between-persons coordination vs. the haptic perceptual system in within-person coordination). The uniqueness of the between-persons situation is that the control structure for coordination must occur over both nervous systems that are necessarily linked by perception. As such, we test not only if a dynamical control structure exists in between-persons coordination, but also the claim that the interaction of an organism with a dynamical control structure used to coordinate its movements (the formation and tuning of the control structure) is based on perception. The question addressed here is whether perception is a process that sustains the dynamical coordination of the activities of two perceivers; that is, whether perceptual information specific to the dynamical landscape of phasing of limbs exists so that it can harness the phasing of the limbs. That this is possible in visual perception is supported by visual perception studies that have shown that dynamical properties such as mass (Bingham, 1987; Runeson \& Frykholm, 1981), momentum (Todd \& Warren, 1982), and the amount of energy about to be exerted (Warren, 1984) can be perceived on the basis of kinematic and geometric properties in the optical structure created by events.

\section{Experiment 1}

Experiment 1 investigates whether the general characteristic properties of the two modes of limb phasing in within-person coordination also exists in between-persons coordination. Two seated subjects were asked to coordinate visually their lower legs either in symmetric phase mode or alternate phase mode while oscillating them at a tempo dictated by an auditory metronome pulse. Of interest is the relative stability of the two modes of phasing in the between-persons situation: whether the magnitude of the fluctuations in relative phase angle and the likelihood of a breakdown in phase locking of the two phase modes grow as the frequency of oscillation is increased.

\section{Method}

Subjects. Twelve undergraduates, 10 women and 2 men, from the University of Connecticut served as subjects in partial fulfillment of a course requirement. They were between the ages of 18 and 23, and had no physical disabilities of any kind. The subjects signed up for the experiment in pairs and may or may not have known each other. Because movements were coordinated between the 2 subjects, the 2 subjects constitute what is called a subject pair (SP). There were, then, six subject pairs: five with 2 female and one with 2 male subjects.

Materials and procedure. Subjects sat on stools $1 \mathrm{~m}$ in height, approximately $1.5 \mathrm{~m}$ from each other, and facing the same direction but turned slightly (about $20^{\circ}$ ) toward each other (Figure 1). Each subject swung the lower part of his or her outer leg. The stools were modified with a padded plank of wood that protruded $10 \mathrm{~cm}$ from the outside corner of the stool to support the leg underneath the knee joint. This allowed for comfortable and free movement of the lower part of the designated leg in the anterior/posterior plane-the left leg for the person seated on the left and the right leg for the person seated on the right. A metronome tape was devised that consisted of eight
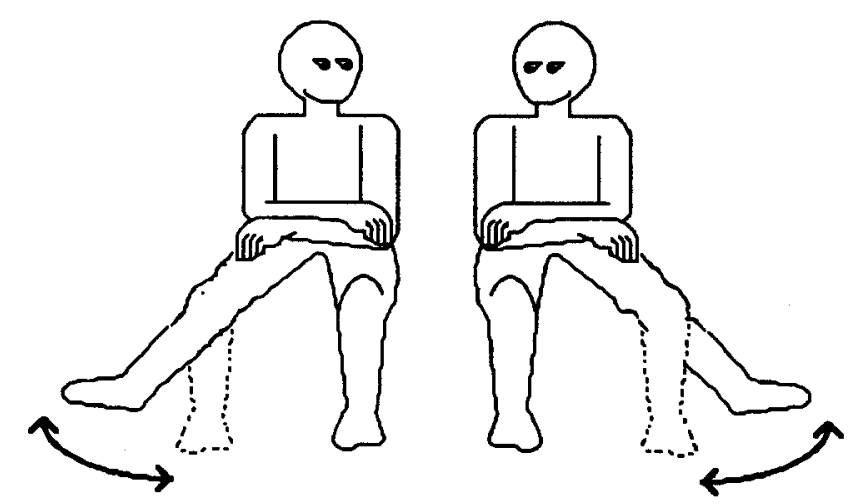

Figure 1. Seating arrangement of the subjects for the experiments. 
frequencies from $0.6 \mathrm{~Hz}$ to $2.0 \mathrm{~Hz}$ at $0.2-\mathrm{Hz}$ intervals. Each frequency lasted $5 \mathrm{~s}$ before the next one began. Leg displacements were recorded by a Panasonic Camcorder PV-320 that was oriented perpendicular to the leg movements $2 \mathrm{~m}$ from the side of the subject on the right (approximately $3.5 \mathrm{~m}$ from the subject on the left).

The experimental session began with a short introduction about between-subjects coordination. Next, the experimenter demonstrated the limb movement required by the task. Subjects were asked to swing the appropriate leg at the frequency dictated by the metronome pulse and consciously coordinated their movements in either symmetric or alternate phase modes relative to the other person's movements. Symmetric mode was described to them as coordination in which their limbs were at the same place in a cycle at the same time. Alternate mode was described as coordination in which their limbs were at opposite places in a cycle at the same time. The two modes of phasing the limbs were also demonstrated with hand motions. After this, one trial from the metronome tape was played for the subjects to complete their familiarization with the details of the task.

All subject pairs were required to coordinate their movements in both phase modes. Most subject pairs found both of the coordinative modes natural and comfortable to maintain at initial tempos, although one subject pair had trouble coordinating initially in the alternate phase mode. The subjects were told that phasing of the limbs may become more difficult as the frequency of oscillation increased. In that case, the subjects were told to keep the frequency of oscillation in pace with the metronome pulse and to try to keep the original phase mode. The three trials of each phase mode condition were blocked. One or two practice trials were performed at the beginning of a phase mode trial block to ensure that the subject pair was performing the task adequately. The order that each subject pair received the two mode conditions was alternated.

Data reduction. The video data records were dubbed from a 0.5 in. videotape to a 0.75 -in videotape so that a Sony U-matic video editor could be used to do a frame-by-frame analysis of the relative phase angle between the limbs. This analysis allowed us to record the time of each peak extension of each subject's leg. The units of time were the reciprocal of the video frame rate, namely, $33.3 \mathrm{~ms}$. The beginning of the trial was arbitrarily designated as the first leg extension of the left subject. From the peak extension times, the frequency of each subject for the $n^{\text {th }}$ cycle was calculated as

$\mathrm{f}_{n}=\left(\right.$ time of peak extension $n-$ time of peak extension $\left.n_{n+1}\right)$, and the relative phase for the $n^{\text {th }}$ cycle as

$\Phi_{n}=360^{\circ}\left[\left(\right.\right.$ peak extension ${ }_{1 n}-$ peak extension $\left.{ }_{2 n}\right) /\left(\right.$ peak extension $_{1 n}$ - peak extension $1 n+1)]$,

following Yaminishi, Kawato, and Suzuki (1979); $1 n$ refers to the $n^{\text {th }}$ cycle of the subject on the right and $2 n$ refers to the $n^{\text {th }}$ cycle of the subject on the left. The phase values were normalized to index the deviation from the steady phase relations at $0^{\circ}$ and $180^{\circ}$ for the symmetric and alternate modes, respectively. Phase values greater than $180^{\circ}$ were normalized by the transform $180-|\Phi-180|$, and phase values less than $0^{\circ}$ were normalized by the transform $|\Phi-0|$. The relative phase values $(\Phi)$ referred to in this article are the normalized values.

\section{Results and Discussion}

After the initial practice trials, the subjects tracked the metronome pulse with relative ease. When asked which of the phase modes they found harder to maintain, they overwhelmingly answered that it was the alternate mode. Although breakdowns in the phasing occurred in both modes, it oc- curred more often in the alternate mode than in the symmetric mode. Sometimes these breakdowns led to a settling into the other phase mode. Because the subjects were instructed to maintain the original phase mode, these transitions to the other phase mode were always temporary and were followed by an attempt to return to the original phase mode.

To analyze the relative stability of the phasing in the two modes, two dependent variables were used, the standard deviation of relative phase and the mean deviation from intended phase ( $\Phi$ for the symmetric mode and $180-\Phi$ for the alternate mode). These two dependent variables were calculated over the cycles at each of the eight frequencies of oscillation. Because the observed frequencies were generally some small deviation from metronome-specified frequencies, the cycles that fell in a frequency range corresponding to half way to the next metronome frequency (metronome frequen$\mathrm{cy}_{n} \pm \mid$ metronome frequency $y_{n}-$ metronome frequency $\left._{n+1} \mid\right)$ were used to calculate the mean deviation from the intended phase and the standard deviation of the relative phase values.

The standard deviations of the relative phase at each metronome frequency are plotted in Figure 2. At the lower frequencies of oscillation, the standard deviations of the relative phase angle are about equal in magnitude for the two phase modes. At higher frequencies, the fluctuation in relative phase for the two modes diverge: The alternate mode increases at a faster rate than the symmetric mode. The standard deviations reach a maximum of $47.9^{\circ}$ at $2.0 \mathrm{~Hz}$ for the alternate mode and a maximum of $28.9^{\circ}$ at $1.8 \mathrm{~Hz}$ for the symmetric mode. A two-way analysis of variance (ANOVA) with within-subject variables of phase mode (symmetric and alternate) and metronome frequency $(0.6,0.8,1.0,1.2,1.4$, $1.6,1.8$, and $2.0 \mathrm{~Hz}$ ) yielded a significant interaction of phase mode by metronome frequency, $F(7,35)=3.37, M S_{\mathrm{e}}=.001$, $p<.01$. Simple-effects tests of this interaction indicate that the fluctuation in the two phase modes differed significantly

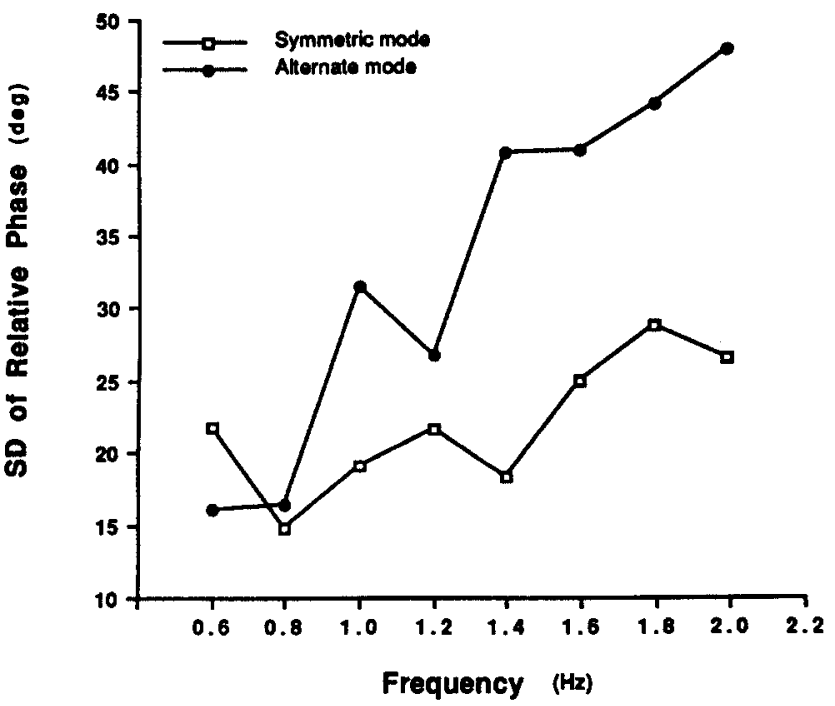

Figure 2. Standard deviation of relative phase at each frequency of the pacing metronome for the two phase modes, symmetric and alternate, in Experiment 1. (Open squares are symmetric mode; closed circles are alternate mode.) 
at the four highest frequencies of oscillation (at $1.4 \mathrm{~Hz}: F[1$, $5]=50.877, M S_{\mathrm{e}}<.001, p<.001$; at $1.6 \mathrm{~Hz}: F[1,5]=9.069$, $M S_{\mathrm{e}}=.001, p<.05 ;$ at $1.8 \mathrm{~Hz}: F[1,5]=17.716, M S_{\mathrm{e}}<$ $.001, p<.01$; and at $2.0 \mathrm{~Hz}: F[1,5]=7.71, M S_{\mathrm{e}}=.001, p<$ .05 ) but not at the lower frequencies (at $0.6 \mathrm{~Hz}: F[1,5]=$ $0.759, M S_{\mathrm{e}}=.001, p>.05$; at $0.8 \mathrm{~Hz}: F[1,5]=0.505, M S_{\mathrm{e}}$ $<.001, p>.05$; at $1.0 \mathrm{~Hz}: F[1,5]=2.006, M S_{\mathrm{e}}=.002, p>$ .05 ; and at $1.2 \mathrm{~Hz}: F[1,5]=2.967, M S_{\mathrm{e}}<.001, p>.05$ ). These results point to a difference in the stability of the two phase modes at the higher frequencies of oscillation.

Further tests of simple effects at each phase mode were performed separately and revealed a significant increase in the fluctuations of relative phase for the alternate phase mode, $F(7,35)=10.125, M S_{\mathrm{e}}=.001, p<.001$, but not for the symmetric phase mode, $F(7,35)=1.149, M S_{\mathrm{e}}=.001, p>$ .05 . This analysis indicates that the stability of the symmetric phase mode remains constant but the alternate mode becomes progressively less stable with increases in the frequency of oscillation.

How well the subjects maintained the intended relative phase angle for the two modes of phasing can be seen in Figure 3, in which the mean deviation from the intended phase is plotted against the metronome frequency. A two-way ANOVA with within-subject variables of phase mode (symmetric and alternate) and metronome frequency $(0.6,0.8,1.0$, $1.2,1.4,1.6,1.8$, and $2.0 \mathrm{~Hz}$ ) performed on these data revealed that the deviation from the intended phase became greater as the frequency of oscillation increased, $F(7,35)=6.785$, $M S_{\mathrm{e}}=.003, p<.001$. More important, the analysis indicated that the deviation from the intended phase was different for the two phase modes, $F(1,5)=19.209, M S_{\mathrm{e}}=.007, p<.01$, with the deviation being greater for the alternate mode $\left(61.02^{\circ}\right)$ than the symmetric mode $\left(34.20^{\circ}\right)$. The Phase Mode $\times$ Metronome Frequency interaction was not significant, $F(7$, $35)=.983, M S_{\mathrm{e}}=.063, p>.05$.

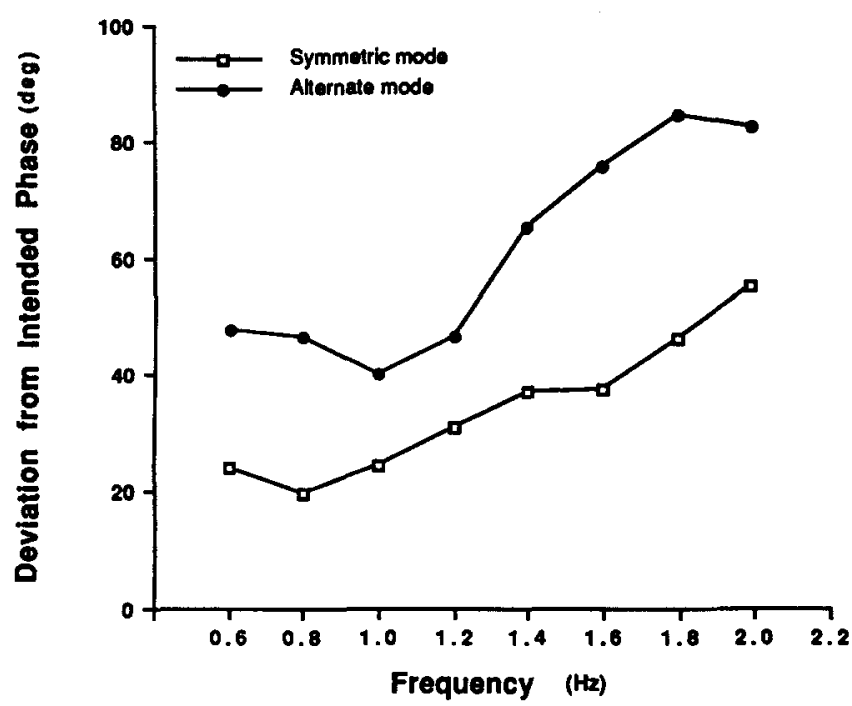

Figure 3. Deviation from intended relative phase at each frequency of the pacing metronome for the two phase modes, symmetric and alternate, in Experiment 1. (Open squares are symmetric mode; closed circles are alternate mode.)
Although this interaction is not significant, the differences in the magnitudes of the deviations in the two phase modes indicate qualitative differences in the phase locking that occurs in the two modes. Studies of the steady state phasing of pendulums held and swung in the right and left hands, in which the pendulums differ in the magnitudes of their dimensions, have revealed a maximum deviation from the intended phase of approximately $50^{\circ}$ (Rosenblum \& Turvey, 1988). Studies of the transition from the alternate mode to the symmetric mode of phasing in within-person bimanual coordination have noted deviations from the intended phase at the transition point of approximately $90^{\circ}$ (Kelso et al., 1986). This value occurs when phase locking breaks down completely. In fact, it must occur when two oscillators are not phase locked at all but are moving continually from one relative phase angle to another. The symmetric mode mean deviations from the intended phase are all at or below the $50^{\circ}$ expected of steady state relative phase angles. However, the alternate mode mean deviations from the intended phase for the last four metronome frequencies are all above the $50^{\circ}$ observed in steady state and are approaching the value $\left(90^{\circ}\right)$ observed in a breakdown of phase locking $\left(1.4 \mathrm{~Hz}: 65.1^{\circ}, 1.6\right.$ $\mathrm{Hz}: 75.6^{\circ}, 1.8 \mathrm{~Hz}: 84.3^{\circ}$, and $\left.2.0 \mathrm{~Hz}: 82.5^{\circ}\right)$. Inspection of the time series reveals that there are many periods at higher frequencies within the alternate mode trials in which a steady relative phase angle is not maintained.

In order to investigate the nonsteady state aspects of the mean deviations from the intended phase, an analysis of the mean number of nonsteady state cycles per trial was performed. The nonsteady state cycles were separated into three groups corresponding to the nonsteady state regions of the relative phase (Figure 4) to which they belong. The relative phase space has one steady state region for each phase mode (from $0^{\circ}$ to $50^{\circ}$ for the symmetric mode and from $135^{\circ}$ to $180^{\circ}$ for the alternate mode) and three nonsteady-state regions for each phase mode: one from the steady state boundary $\left(50^{\circ}\right.$ for symmetric mode or $135^{\circ}$ for alternate mode) to the transition point $\left(90^{\circ}\right)$, another from the transition point to the steady state boundary of the other mode $\left(135^{\circ}\right.$ for symmetric mode or $50^{\circ}$ for alternate mode), and the last from the steady state region of the other phase mode. These nonsteady state regions will be denoted as NSR 1, NSR 2, and NSR 3 (Nonsteady State Regions 1, 2, and 3), respectively. A threeway ANOVA with within-subject variables of phase mode (symmetric and alternate), region (NSR 1, NSR 2, and NSR 3), and metronome frequency $(0.6,0.8,1.0,1.2,1.4,1.6,1.8$, and $2.0 \mathrm{~Hz}$ ) was performed on the mean number of transitory cycles per trial. A significant three-way Phase Mode $\times$ Region $x$ Metronome Frequency interaction was found, suggesting that the relationship of phase mode and metronome frequency was different for the nonsteady state cycles in the different regions, $F(14,70)=2.011, M S_{\mathrm{e}}=.475, p<.05$. Simpleeffects tests of this interaction at each of the three different nonsteady state regions revealed significant Phase $\times$ Metronome Frequency interactions for NSR $2, F(7,35)=3.584$, $M S_{\mathrm{e}}=.283, p<.01$, and NSR 3, $F(7,35)=2.710, M S_{\mathrm{e}}=$ $.727, p<.05$, but for NSR 1 , only a significant main effect of frequency was found, $F(7,35)=3.959, M S_{\mathrm{e}}=.773, p<.01$ (Figure 5). These analyses indicate that the differences in the 


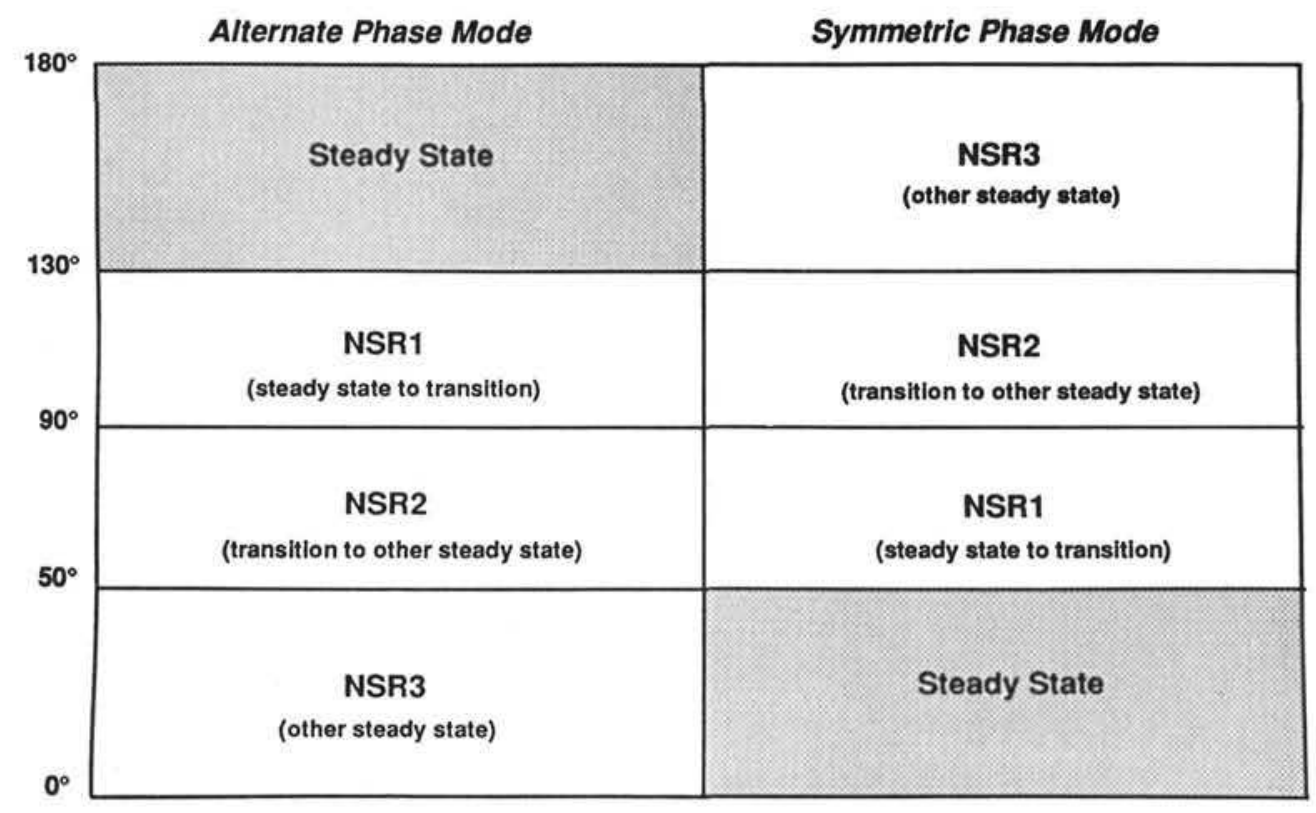

Figure 4. Steady and nonsteady regions of the relative phase space.

stabilities of the two phase modes occur in terms of the number of nonsteady state cycles that are past the transition point to the other mode, that is, the number of cycles of broken phase locking that are indicative of transition behavior. The alternate phase mode has a greater number of nonsteady state cycles that are past the transition point to the symmetric phase mode, and the number of these cycles increase with the frequency of oscillation. This can be taken as preliminary evidence that a bifurcation phenomenon could occur in visually coupled limbs analogous to that observed in gait transitions and within-person bimanual coordination. Experiment 2 investigates this possibility using the same task with different instructions.

\section{Experiment 2}

Experiment 1 provided evidence that some of the properties that exist in the two modes of limb phasing in within-person coordination also exist in the two modes of limb phasing in between-persons coordination. In the within-person phasing of limbs, the alternate phase mode grows less stable as the frequency of oscillation increases, whereas the symmetric mode remains relatively stable at all frequencies. The same pattern was observed in Experiment 1 in the between-persons phasing of limbs using the standard deviation and mean deviation from the intended phase as indices of stability. Furthermore, in the within-person phasing of limbs, the growing instability of the alternate mode leads to a breakdown of phase locking followed by a transition to the symmetric mode of phasing. Preliminary evidence for a breakdown of the alternate mode phasing in between-persons coordination was found in Experiment 1: More cycles were observed beyond the transition point and in the other phase mode for the alternate mode than for the symmetric mode trials at high metronome frequencies. Experiment 2 investigates whether the between-persons coordination task used in the Experiment 1 can be constrained to demonstrate the transition proper from the alternate phase mode to the symmetric phase mode (but not vice versa) as in between-persons coordination. A positive outcome would favor the hypothesis that the control structure that harnesses the coordination between two people is the same dynamical control structure that is operating in the coordination of limbs within a person.

In Experiment 1, subjects were instructed to oscillate the lower part of their legs at the same frequency and remain in the initial mode of phasing. Given these constraints, the intention of each subject of a subject pair after a breakdown in phase locking was to try to return to the initial phase mode even if it meant not being phase locked at all. Indeed, the data of Experiment 1 demonstrate that the subjects spent some time in neither phase mode in the alternate mode trials. In Experiment 2, the task demands were changed. The subjects were again asked to oscillate their legs at a common frequency and to begin in either of the two phase modes. However, the subjects were told that if a breakdown occurred in the phasing of the limbs, they were to remain in the new phase mode if they found it more stable than the initial phase mode. Compared with Experiment 1, these demands are more analogous to those in the experiments that demonstrated the within-person bimanual phase transition (Scholz, 1986; and, presumably, are more analogous to the tacit intentions of gait transitions).

\section{Method}

Subjects. A 27-year-old male graduate student (LR) and a 33-year-old female professor (CC), both associated with the University of Connecticut and Haskins Laboratories, participated in the experiment. Both were right-handed, and neither had motor disabilities of any kind. 

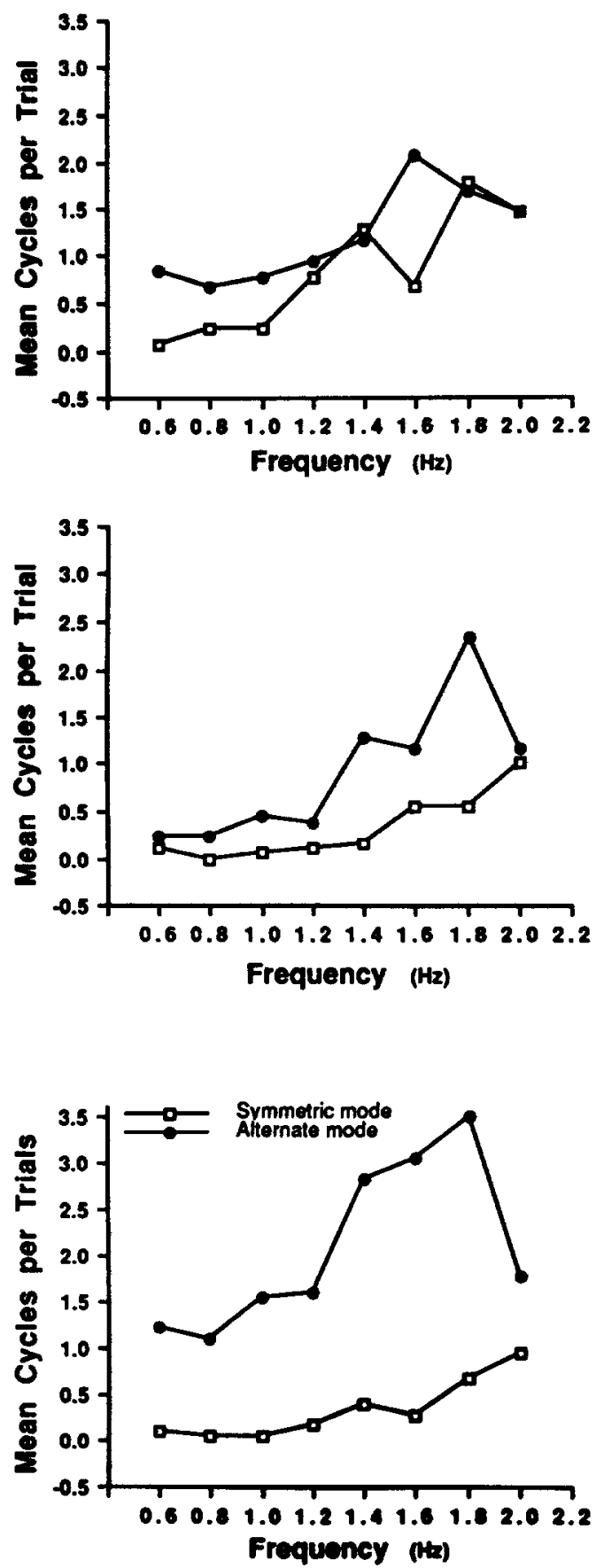

Figure 5. Mean number of nonsteady cycles per trial at each frequency of the pacing metronome for the two phase modes, symmetric and alternate. (The top panel is the mean number of nonsteady cycles found in the steady state boundary to the transition point $\left(90^{\circ}\right)$ region. The middle panel is the mean number of nonsteady cycles found from the transition point to the steady state boundary of the other mode. The bottom panel is the mean number of nonsteady cycles found in the steady state region of the other phase mode.)

Materials and procedure. The same experimental arrangement was used as in Experiment 1. LR oscillated his lower left leg and CC oscillated her lower right leg (Figure 1) at the same frequency. Symmetric mode was described to them as coordination in which their limbs were at the same place in a cycle at the same time. Alternate mode was described as coordination in which their limbs were at opposite places in a cycle at the same time. The subjects found both of these coordinative modes natural and comfortable to maintain at initial tempos. Leg displacements were measured by a Teka PN-4 Polgon goniometer. Polarized light sensors were attached $10 \mathrm{~cm}$ above the knee and $12 \mathrm{~cm}$ below the knee of each subjects' outer leg. The data were recorded onto FMmagnetic tape and later digitized at 200 samples/s using a Datel ST-PDP 12 bit analogue-to-digital converter.

The simplest version of the phase transition methodology was used in which the experimenter determined when the frequency was to be increased, but the subjects determined how much the frequency was to be increased (Kelso, 1984; Kelso et al., 1986). After $10 \mathrm{~s}$ of a mutually comfortable tempo in either phase mode, the subjects were asked to increase the movement frequency slightly. Every $10 \mathrm{~s}$ thereafter, the experimenter signaled another frequency increase. Subjects were instructed to maintain the initial phase mode as the oscillation frequency increased. It was suggested, however, that maintaining the initial phase-locked state might become difficult and that switching to another phase mode was allowed (and might occur). If this new phase mode was more stable, they were instructed to remain in it. A total of 10 trials for initial alternate mode and 5 trials for the initial symmetric mode were performed on 2 separate days. The alternate mode trials were collected on the 1st day and the symmetric mode trials were collected on the 2 nd day. The trials of a condition were blocked together.

Data reduction. The digitized data records were smoothed using a Bartlett (triangular) moving average procedure with a window size of $35 \mathrm{~ms}$. Software analysis routines determined the frequency of oscillation of each subject's leg for each cycle and the normalized relative phase between the legs of the 2 subjects using the formulas presented in Experiment 1.

\section{Results}

The subjects had three goals in these trials: to oscillate their legs at a common frequency, to increase this frequency of oscillation steadily, and to phase their limbs initially in the alternate or symmetric phase mode while trying to keep this phasing comfortable. Each of these goals will be evaluated in turn.

Degree of synchrony. The degree of synchrony between the 2 subjects can be evaluated through a regression of the cycle frequencies of one subject on the cycle frequencies of the other subject. For a system in a state of perfect synchrony, such a regression analysis would reveal a slope of unity, an intercept of zero, and an $r^{2}$ of unity. The results of such an analysis on the inverse of frequency (period) for both phase modes are presented in Figure 6. For symmetric and altemate modes, respectively, the slopes are effectively unity $(0.992$ and 1.000$)$, the intercepts are near zero $(0.003$ and -.019$)$, and the $r^{2}$ s are near one $\left(r^{2}=.969, F[1,269]=8354.451\right.$, $p<.001$, and $r^{2}=.909, F[1,318]=3161.182, p<.001$.

Rate of frequency increase. The frequency ranged from $0.694 \mathrm{~Hz}$ to $2.381 \mathrm{~Hz}$ in the symmetric mode condition and 

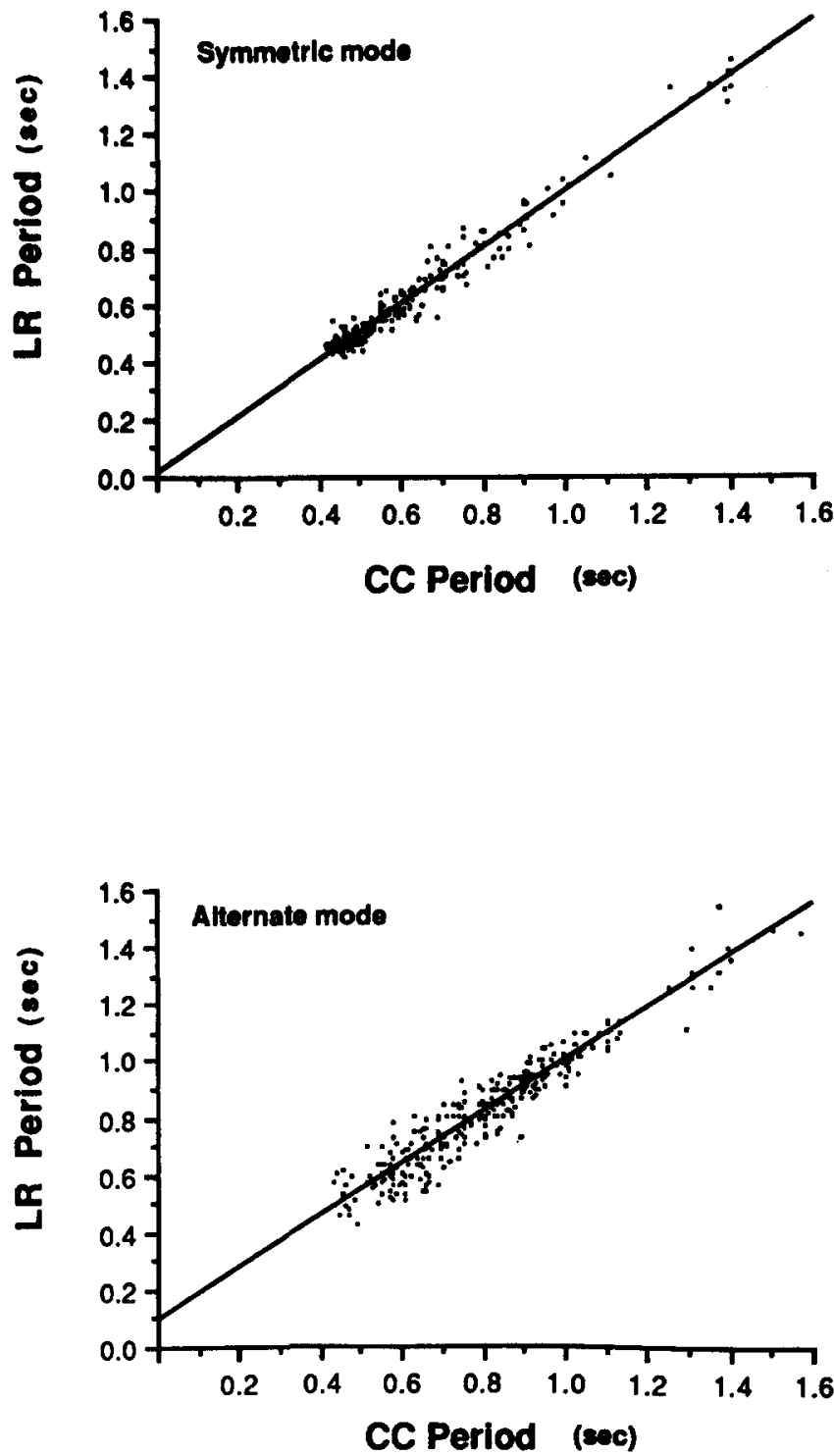

Figure 6. Regression of period of subject $\mathrm{CC}$ on period of subject LR in Experiment 2.

from $0.637 \mathrm{~Hz}$ to $2.326 \mathrm{~Hz}$ in the alternate mode condition. Regression analysis can be used to evaluate whether the rate of frequency increase across the cycles of a trial was uniform for the two modes of coordination. In the regression of mean frequency of the 2 subjects on cycle number, the slope is an index of the speed of the increase of the entrainment frequency, the intercept is an index of the initial frequency, and the $r^{2}$ is an index of how steadily an increase was maintained. The $r^{2}$ s are expected to be less than unity because the instructions demand a frequency increase once every $10 \mathrm{~s}$. Significant linear regressions were obtained for all trials in both conditions, indicating that the subjects were increasing the frequency of oscillation steadily within a trial. The rate of frequency increase became greater after the second trial of the alternate mode condition; hence, regressions were performed on the first two and last eight alternate mode trials separately.
Pooled trials for each condition yielded regression equations of $0.022 X+1.141$, with $r^{2}=.748, F(1,269)=799.169, p<$ .001 , for the symmetric mode; and $0.012 X+.765$, with $r^{2}=$ $.771, F(1,82)=275.770, p<.001$, for first two trials; and $0.030 X+.895, r^{2}=.856, F(1,234)=1396.672, p<.001$, for the last eight trials of the alternate mode. A statistical comparison of the regression equations for the symmetric mode and the last eight trials of the alternate mode found the equations to differ significantly in their intercepts but not their slopes, $t(1,504)=6.242, p<.001$. This result indicates that the rate of frequency change (i.e., the slope) was uniform across the two experimental conditions, but that the initial frequency deemed comfortable by the 2 subjects was higher for the symmetric mode $(1.141 \mathrm{~Hz})$ than for the alternate mode $(0.895 \mathrm{~Hz})$.

Relative phase. The subjects were to maintain a phaselocked state in either the symmetric or alternate phase mode as the frequency of oscillation increased. They reported that maintaining a steady and comfortable phasing was easy at all frequencies in the symmetric mode and at the lower frequencies in the alternate mode. However, at some point in the alternate mode phasing, the phase locking would break down and thereafter only the symmetric mode was comfortable to maintain. This occurred in all 10 recorded trials. Typical position and relative phase time series for both the mode conditions are portrayed in Figure 7.

To summarize phase locking across the trials of a condition, we broke down each trial into segments containing $0.2-\mathrm{Hz}$ frequency steps in a range from $0.6 \mathrm{~Hz}$ to $2.0 \mathrm{~Hz}$. For each segment of a trial, the mean frequency and the mean relative phase were calculated from cycles that fell in that particular $0.2-\mathrm{Hz}$ frequency range. Summary means of the relative phase and frequency for each frequency segment of the symmetric mode condition, calculated across all trials, are plotted against each other in Figure 8 (upper panel). The range of mean relative phase exhibited across the frequencies was between $12^{\circ}$ and $25^{\circ}$. By the criteria mentioned in Experiment 1, these values indicate steady state phase locking at all frequencies of oscillation.

An identical plot would not be legitimate for the alternate mode trials because the frequency at which the transition occurred was not the same for all trials, ranging from 1.087 $\mathrm{Hz}$ to $1.575 \mathrm{~Hz}$, with a mean of $1.301 \mathrm{~Hz}$ and a standard deviation of 0.167 . Because these transitional frequency values fell into different $0.2-\mathrm{Hz}$ frequency segments, summary means calculated on the basis of segments with the same frequencies would average transitional states with steady states. To remedy this situation, alternate mode trials were aligned so that the frequency segment of the transition was $F_{\mathrm{t}}$ and the frequency segments before and after the transition were $F_{1-\mathrm{i}}$ and $F_{\mathrm{t}+\mathrm{i}}$, respectively, where $i$ is the number of segments that a given segment is from the transition. The mean relative phase was calculated for these segments and averaged across trials. Data points that represented transitional values were eliminated from steady state segments' means. A plot of the mean relative phase aligned in relation to the transition for all the alternate mode trials is presented in Figure 8 (lower panel). There is a sudden jump in the alternate mode trials from the relative phase values that are 

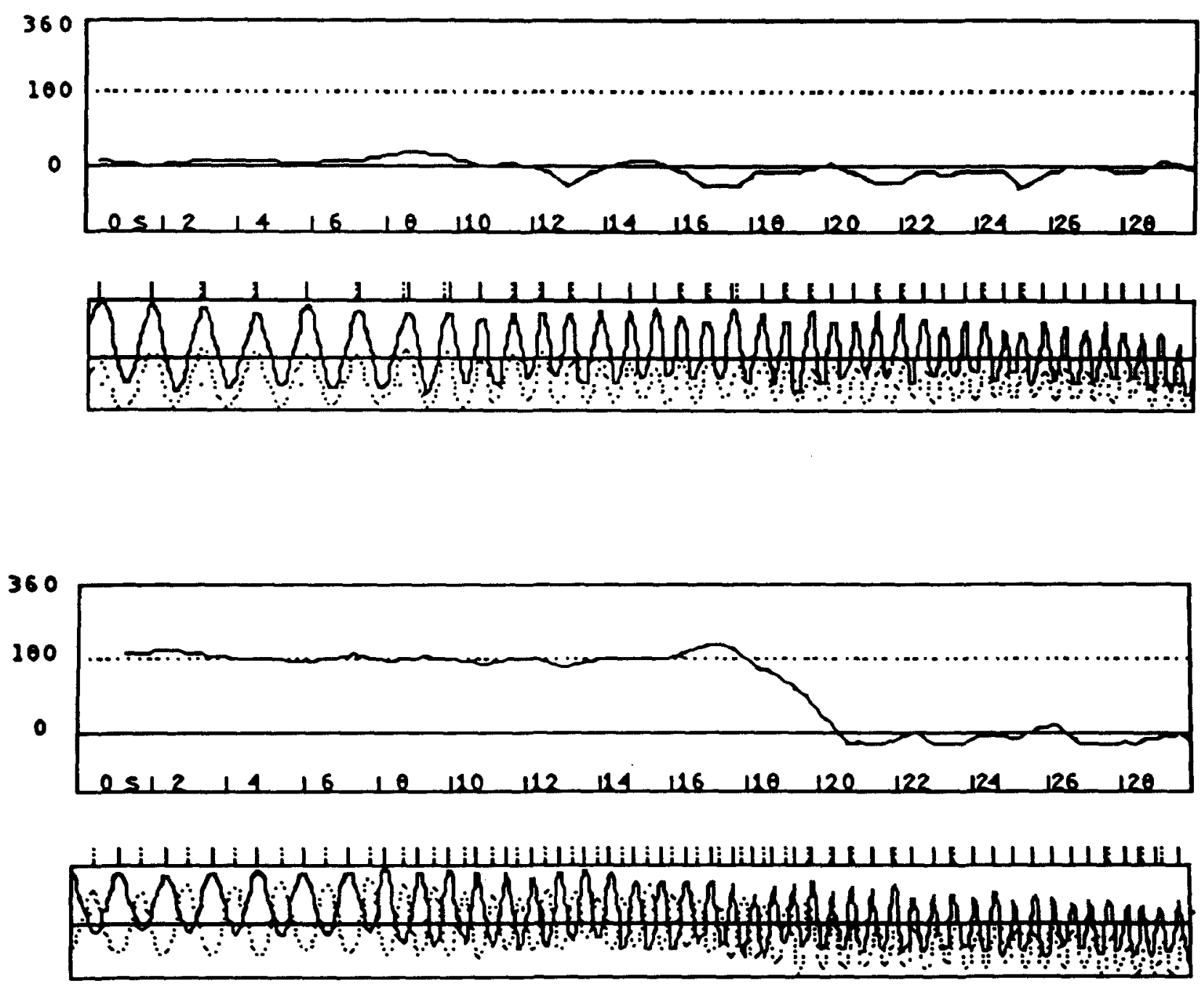

Figure 7. Relative phase and position time series for the symmetric mode (upper pair of panels) and the alternate mode (lower pair) in Experiment 2. (The vertical axis for the upper panel in a pair is the phase relation between the two limbs in degrees; for the lower panel it is the amplitude of the rhythmic movement. The horizontal axis for both panels is time in seconds.)

characteristic of steady state alternate mode phasing $(\Phi>$ $130)$ to those that are characteristic of steady state symmetric mode phasing $(\Phi<50)$, with a transitional nonsteady state phase value in between $(\Phi \approx 83)$.

The similarities between the characteristics of the two phase modes performed between the limbs of a single person and the limbs of two people are quite remarkable. In both cases, the stability of the alternate mode decreases dramatically relative to the stability of the symmetric mode as the frequency of oscillation increases. Furthermore, under the proper task constraints, in both cases, this decrease in stability of the alternate mode is seen to precipitate a jump from this mode to the symmetric phase mode. Given these similarities, Experiment 3 will test whether the control structure underlying the between-persons phasing of limbs exhibits a dynamical organization identical with that of the control structure underlying the within-person phasing of limbs.

\section{Experiment 3}

Haken et al. (1985) modeled the sudden jump from the alternate mode to the symmetric mode as a physical bifurcation. This kind of nonlinear reordering is found rather generically in physical systems. It occurs in a physical system when the attractor governing the system's behavior is made suddenly unstable by virtue of continuous scaling of some control parameter (Gilmore, 1981). The phenomenological mode of the attractor layout underlying the transition from the alternate to the symmetric phase mode proposed by Haken et al. interprets the alternate and symmetric coordinations as point attractors found at the minima of the potential function

$$
V(\Phi)=-a \cos (\Phi)-b \cos (2 \Phi) \text {. }
$$

This potential function satisfies symmetry requirements of the phenomenon and is derivable from the equations of 

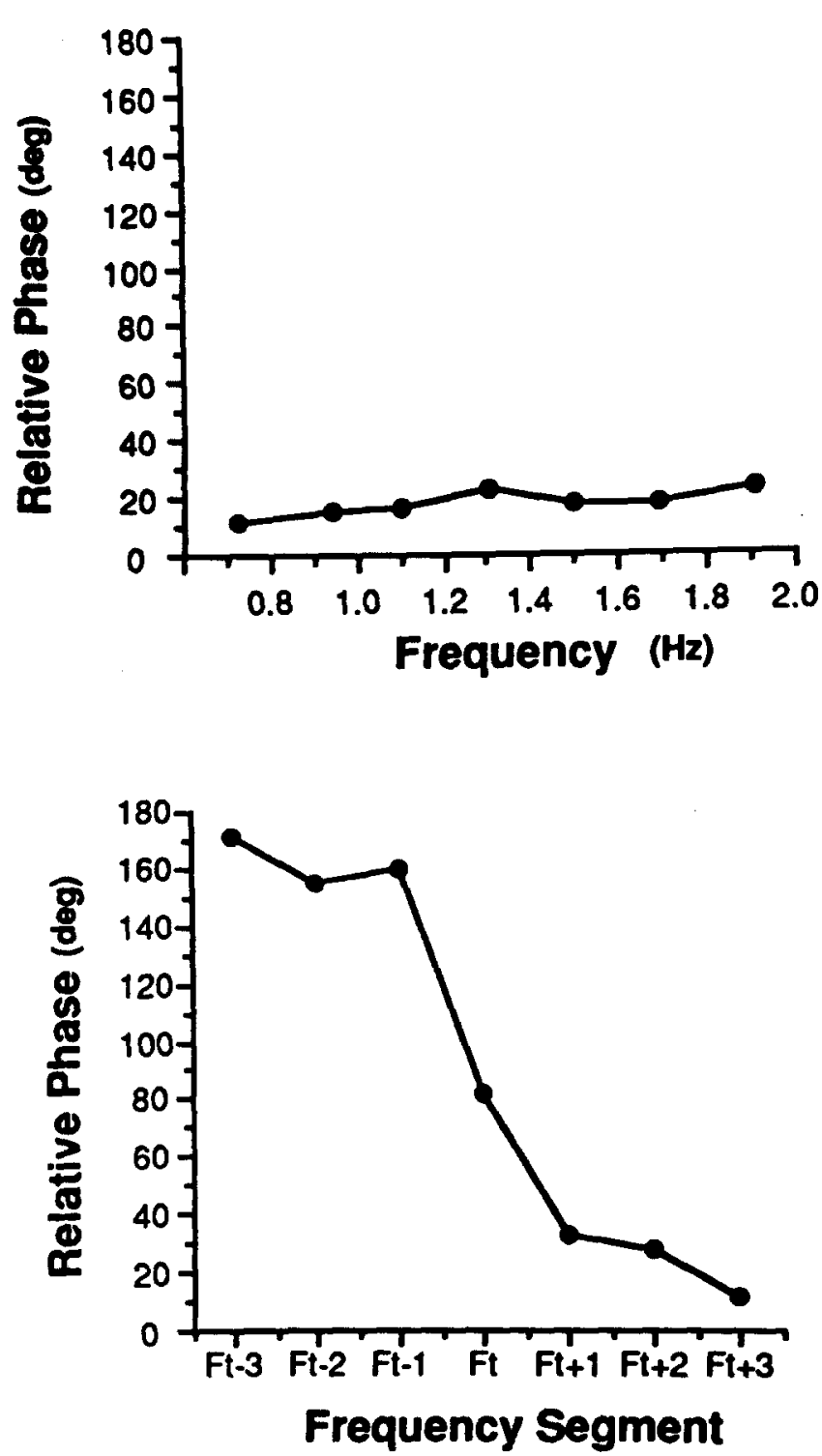

Figure 8. Relative phase as a function of mean frequency for each frequency segment in the symmetric mode (upper panel); relative phase as a function of frequency scaled relative to the transition frequency for the alternate mode (lower panel).

motion of the component oscillators. Following the basic ideas of synergetics-a school of thought dealing with cooperative phenomena generally (Haken, 1978, 1983)-the relative phase $\Phi$ is identified as an order parameter (i.e., a quantity representing the macroscopic ordering of component parts) with relaxational dynamics

$$
\mathrm{d} \Phi / \mathrm{d} t=\partial V / \partial \Phi .
$$

For $b / a<.25, \Phi=0^{\circ}$ and $\Phi= \pm 180^{\circ}$ are minima; at $b / a=$ .25 , the local minima at $\pm 180^{\circ}$ become unstable. Haken et al. provide an account of how the preceding potential function can be derived from component oscillator equations with a nonlinear coupling. Computer simulations demonstrate that the function mimics the observed bifurcation as the frequency is scaled.
A stochastic generalization of the analysis (Schöner et al., 1986) predicts important dynamical accompaniments of the destabilization leading to a phase transition, namely, an increase in fluctuations in relative phase and an increase in the time to return from a perturbation to a steady state trajectory (known as relaxation time). Empirical studies have affirmed these predictions. Kelso and Scholz (1985) paced the movement frequency with a metronome pulse that increased from 1.25 to $3.0 \mathrm{~Hz}$ in $0.25-\mathrm{Hz}$ increments every $4 \mathrm{~s}$. Fluctuations in phase (standard deviation of $\Phi$ ) were found to increase as the transition was approached and decreased to minimal values after the symmetric mode was attained. Because the system before the transition is approaching a critical point, the state of the system is characterized by increasing criticality and the fluctuations observed are referred to as critical fluctuations.

The existence of critical fluctuations before the transition was replicated in two separate experiments (Kelso et al., 1986). However, in a later experiment in which the amount of time the oscillation remained at a specific frequency was increased from $4 \mathrm{~s}$ to $10 \mathrm{~s}$, critical fluctuations were not observed (Scholz, 1986; Scholz et al., 1987). These results indicate that the bifurcation from the alternate to the symmetric mode occurs in this case before the potential minimum at $180^{\circ}$ becomes very unstable. This is to be expected when the frequency plateau time is not much less than the time needed for a change from the minimum at $180^{\circ}$ to the minimum at $0^{\circ}$ to occur probabilistically (i.e., the "random walk" time or the first passage time; Gilmore, 1979, 1981; Scholz et al., 1987).

Hence, whether critical fluctuations are observed before a bifurcation depends on two different states of affairs: Either the system remains in the first mode until the attractor is completely annihilated and critical fluctuations are observed, or a stochastic perturbation knocks the system into the second mode before the attractor is completely destroyed and critical fluctuations are not observed. These two different states of affairs are called the delay convention and the Maxwell convention, respectively, by some theorists (e.g., Gilmore, 1979, 1981). Other theorists discuss the difference in terms of the relationship of different time scales operating in the phenomenon (Schöner et al., 1986). The delay convention holds if the fast time scale $\tau_{\text {relaxation associated with the relaxation back }}$ to the alternate phase mode is notably less than the time scale $\tau_{\text {control }}$ of change in the control parameter, which, in turn, is notably less than the slow time scale $\tau_{\text {passage }}$ associated with the first (probabilistic) passage from the alternate to the symmetric phase mode (i.e., $\tau_{\text {relaxation }} \ll \tau_{\text {control }} \ll \tau_{\text {passage }}$ ). In this case, there is a change of coordination only when the old coordination becomes unstable and when critical fluctuations are observed. Alternatively, order parameter fluctuations do not anticipate the sudden behavioral change when the time scale of the passage between equilibria is less than the time scale of the control parameter; that is, $\tau_{\text {passage }} \ll \tau_{\text {control. }}$. In this case, the Maxwell convention holds. Under such conditions, the switch between coordinations occurs before the old coordination destabilizes. The practical point of all this is that, in an experiment, conditions should be devised to account for the operation of both possible states of affairs, that is, conditions with different control parameter change times $\left(\tau_{\text {control }}\right)$. 
In summary, Kelso and associates (Kelso et al., 1986; Scholz et al., 1987) have provided evidence that the transition from the alternate to the symmetric phase mode is the result of the increasing instability of the attractor underlying the phase locking of limbs. The characteristic properties of this phase transition are (a) a sudden jump between two modes of functioning that represents, (b) a divergent response to the scaling of frequency, (c) inaccessible regions between the two modes, (d) hysteresis in the transition (slowing down after the transition does not yield a shift back to the alternate mode), (e) critical fluctuations before the transition when the change time is less than the first passage time, and (f) an increase in relaxation time as the transition is approached.

Experiment 3 investigates whether these properties occur in between-persons visual coordination of legs oscillated about the knee joint. An affirmative answer would indicate a dynamical basis for the control structure governing betweenpersons phasing of limbs. The experiment was performed to determine if the sudden jump exhibited criterial properties of a physical bifurcation: divergence, critical fluctuations, and hysteresis. If the spontaneous behavioral discontinuity observed in between-persons coordination has these criterial properties, then the claim can be made that the coordination of movements between people can have a dynamical basis. Furthermore, given the dependence of this kind of coordination on the availability of light structured by and specific to the movements of the limbs, a claim can then be made for the role of information, in Gibson's $(1966,1979)$ specificational sense, in the expression of dynamical principles (Kugler \& Turvey, 1987).

\section{Method}

Subjects. The same 2 subjects from Experiment 2 served as voluntary participants. A third volunteer associated with Haskins Laboratories, a 32-year-old right-handed man (GB) with no motor disabilities was added to permit three possible pairings of the subjects: SP 1 included CC and LR, SP 2 included CC and GB, and SP 3 included LR and GB.

Materials and procedure. The same basic materials and data collection procedure as in Experiment 2 were used. However, as in Experiment 1, a metronome pulse played from a cassette tape over a loudspeaker was used to scale the frequency. Time records of the metronome pulse and lower leg displacement were recorded on FM tape and later digitized at 200 samples/s.

Again, the 2 members of a subject pair were instructed to oscillate the lower part of their legs at the same frequency in either symmetric or alternate phase modes as the frequency of oscillation was increased by the metronome. As in Experiment 1, the metronome pulse remained at a frequency for a given amount of time before it was moved to a frequency $0.2 \mathrm{~Hz}$ greater. The amount of time the pulse remained at a frequency is referred to as the frequency plateau time. Because the stationarity (i.e., absence of linear trends) of the time series produced by the method used in Experiment 2 can be called into question (Kelso et al., 1986), the metronome scaling procedure with its plateaus of steady state frequencies was used to reduce the likelihood that the time series are nonstationary.

Because different results have been found for studies using different frequency plateau lengths (see Scholz, 1986; Scholz et al., 1987), two different frequency plateau times were used, $5 \mathrm{~s}$ and $10 \mathrm{~s}$. For each frequency plateau time, 10 symmetric and 10 alternate mode trials were performed by each subject pair. Each trial began with the subjects' establishing and maintaining a phase-locked state in one of the two phase modes at $0.6 \mathrm{~Hz}$ for twice the frequency plateau time $(10 \mathrm{~s}$ or $20 \mathrm{~s})$. Thereafter, the frequency was increased in increments of $0.2 \mathrm{~Hz}$ every $5 \mathrm{~s}$ or $10 \mathrm{~s}$, depending on the frequency plateau condition, to a final frequency of $1.8 \mathrm{~Hz}$ or $2.0 \mathrm{~Hz}$. The final frequency differed for the two frequency plateau conditions and the different subject pairs.

A hysteresis condition was run for one subject pair (SP 1). In these four trials, the initial coordination in the alternate mode was scaled in frequency as in the 5-s frequency plateau trials. Once the final frequency of $1.8 \mathrm{~Hz}$ was reached, the metronome pulse began to decrease the frequency of oscillation back to $0.6 \mathrm{~Hz}$ in the same way that it was increased. These trials were performed to determine the frequency at which a transition back to alternate mode would occur (once the symmetric mode had been established through bifurcation) when the frequency of oscillation was decreased.

Each subject in a pair was instructed to oscillate his or her lower leg at the frequency of the metronome while coordinating the leg's position in relation to the other person's leg. As in Experiment 1, the subjects were instructed to maintain the initial phase mode as the oscillation frequency increased. Again, we suggested that maintaining the initial phase-locked state might become difficult and that switching to another phase mode might occur. If this alternate phase mode was more stable, subjects were instructed to remain in it. The data were collected in two separate sessions on 2 separate days. The 10-s frequency plateau trials were collected in the first session, and the 5$s$ frequency plateau trials and the hysteresis trials were collected in the second session. Each session was about $1 \mathrm{hr}$ in length. The trials of a condition were always blocked together.

Data reduction. The digitized data records were smoothed using a Bartlett (triangular) moving average procedure with a window size of $35 \mathrm{~ms}$. One trial of SP 1 in the 10-s alternate mode condition was lost because of experimenter error. The cycle frequency was calculated the same way as in Experiment 1. The relative phase was estimated not for each cycle as in Experiments 1 and 2 but for each sample, that is, every $5 \mathrm{~ms}$. The algorithm for this continuous phase analysis (CPA) uses normalized values of position and velocity to determine the phase of each oscillator at each sample, and from these calculates the relative phase between the two oscillators to produce a relative phase time series (see Kelso et al., 1986, for more details). The CPA analysis, however, does not calculate the continuous relative phase estimates for the first $2 \mathrm{~s}$ of the data record, that is, $2 \mathrm{~s}$ from the 0.6 $\mathrm{Hz}$ plateau. For the most part this elimination of data was not a problem because there were still enough observations (600 for 5-s plateaus) left to estimate relative phase for that plateau. As in Experiments 1 and 2, the phase values were normalized to index the deviation from the steady phase relations at $0^{\circ}$ and $180^{\circ}$ for the symmetric and alternate modes, respectively. Again, the relative phase values $(\Phi)$ referred to in this section will be the normalized values.

The beginning and end times of each frequency plateau were determined through interactive graphic displays of the metronome pulse time series. The beginning of a transition was defined as the end of the last ascent to $180^{\circ}$ of relative phase time series in the alternate mode steady state region of the relative phase space. In the alternate mode trials, a plateau was categorized as either pretransitional, transitional, or posttransitional. Software analysis routines then calculated the mean of cycle frequency and the mean and standard deviation of the normalized (see Experiment 1) relative phase for all the observations of a plateau. All of these measures were calculated after the first second to ensure that transients from the frequency change had diminished. The total power of continuous phase was calculated for each frequency plateau again (after the first second) by summing over the squared amplitude of the Fast Fourier Transform (FTT; Press, Flannery, Teukolsky, \& Vetterling, 1988) of the continuous phase time series. Because of the large range of total power magnitudes, harmonic means were used to find the central 
tendency and $\log _{\text {e }}$ transforms were taken. Total power estimates were not found for $0.6-\mathrm{Hz}$ frequency plateaus of the 5 -s plateau length condition because of an insufficient number of observations to perform the FFT routine. Transition transients were eliminated from all pre- and posttransitional plateaus.

\section{Results}

As in Experiment 2, the increase of oscillation frequency led to a breakdown of visual phase locking in all the alternate mode trials of the three subject pairs and a transition to the symmetric mode of phasing. No such breakdown of coordination was seen in the symmetric mode trials ${ }^{2}$. Samples of the continuous relative phase time series for the symmetric and alternate modes are shown in Figure 9. Notice the increased resolution of the relative phase compared with the cycle or point phase measures in Figure 7. This increased resolution shows that relative phase is not static in time and often picks up a cyclic component in the plateau before the transition.

The mean transition frequency was $1.529 \mathrm{~Hz}$ with a standard deviation of $0.225 \mathrm{~Hz}$; the modal transition frequency was $1.6 \mathrm{~Hz}(n=20$ of 60 ). A one-way ANOVA demonstrated that the mean transition frequencies of the two frequency plateau conditions ( $5 \mathrm{~s}: 1.633 \mathrm{~Hz} ; 10 \mathrm{~s}: 1.227 \mathrm{~Hz}$ ) were not statistically different. The times taken for the transition to occur were also measured. These were measured from the end of the last ascent to $180^{\circ}$ to the point at which the relative phase equals $0^{\circ}$ or some steady state relative phase value around $0^{\circ}$ (within $45^{\circ}$ ). The mean transition time overall was $3.34 \mathrm{~s}$ with a standard deviation of $1.59 \mathrm{~s}$. The mean transition times for the $5 \mathrm{~s}$ and $10 \mathrm{~s}(2.61 \mathrm{~s}$ and $4.09 \mathrm{~s}$, respectively) were statistically different, $F(1,2)=51.166, M S_{\mathrm{e}}=.064, p<$ .02 .

Divergence. One of the characteristic properties of a physical bifurcation is a divergent response of the dependent variable to the increasing control parameter. At some point in the continuum of control parameter values, for one unit of control parameter change, many units change of dependent measure should be observed. To test for divergence in the alternate mode trials, we identified two subsets of the total number of trials and analyzed the mean deviation from the intended relative phase $(|180-\Phi|$ for the alternate mode and $\Phi$ for the symmetric mode) for each subset, separately. The first subset contained the means of the alternate mode trials with a 1.4-Hz transition frequency. The second subset contained the means of the alternate mode trials with a $1.6-\mathrm{Hz}$ transition frequency. Together these two subsets constituted $63 \%$ of all the trials. These subsets were analyzed separately so that transient states and steady states would not be combined in averaging. Hence, the resultant means are more representative of the deviation from the intended phase seen in the individual trials.

The two subsets were submitted to three-way ANOVAS with within-subject variables of phase mode, frequency plateau length, and metronome frequency. SP 1 did not have any trials that contained a $1.6-\mathrm{Hz}$ transition frequency for the 10 $s$ plateau condition; therefore, the means of the 2 other subjects' data at each frequency of the alternate mode 10-s plateau trials were used to fill in these empty cells. All the means of the symmetric mode trial data were used in both analyses. The means across the two frequency plateau conditions are displayed in Figure 10 for 1.4- and $1.6-\mathrm{Hz}$ transition frequency data. In both sets of data, the two relative phase modes start out at the same small deviation from the intended phase. At the transition frequency, the deviation from the intended phase diverges for the alternate mode condition to values between $100^{\circ}$ and $110^{\circ}$. With another increase in frequency, the alternate mode trials diverge even further to deviation values $\left(\approx 150^{\circ}\right)$ that indicate that the phasing of the limbs is now in the symmetric mode. In contrast, the symmetric condition's deviation from the intended phase remains small $\left(<30^{\circ}\right)$ across all frequency changes.

The two ANOVAS revealed significant interactions of phase mode and metronome frequency $(1.4-\mathrm{Hz}$ transition trials: $F[6,12]=122.422, M S_{\mathrm{e}}=88.594, p<.001 ; 1.6-\mathrm{Hz}$ transition trials: $\left.F[6,12]=499.886, M S_{c}=17.388, p<.001\right)$. Simple effects analyses demonstrated that these effects were the result of the differences of the alternate mode condition from the symmetric mode condition that began at the transition frequency (phase mode at $1.4 \mathrm{~Hz}: F[1,2]=520.645, M S_{\mathrm{e}}=$ $36.686, p<.01$; phase mode at $1.6 \mathrm{~Hz}: F[1,2]=520.645$, $\left.M S_{\mathrm{e}}=16.667, p<.01\right)$ and continued thereafter. Paired comparisons using Fisher's protected $t$ tests of the deviation from the intended phase at the transition frequency with the deviation from the intended phase at the pretransition frequency found the divergence at the transition statistically significant (1.4-Hz transition trials: $p<.01 ; 1.6 \mathrm{~Hz}$-transition trials: $p<.05$ ). A significant three-way interaction between phase mode, frequency plateau length, and metronome frequency was found in the $1.6-\mathrm{Hz}$ data, $F(3,12)=3.202, M S_{\mathrm{e}}$ $=26.309, p<.05$, that can be attributed to the difference in the alternate mode deviation from the intended phase at the transition for the 5-s and 10-s plateaus.

Critical fluctuations measured by standard deviation. All the standard deviations of the relative phase for a given frequency were averaged, keeping pre- and posttransitional plateaus separate, and eliminating transitional plateaus. Because the pretransition frequency range differed between subjects, and differed within a subject for the different frequency plateau lengths, the means were calculated according to where in the range of frequencies they fell. Four frequency categories were chosen: three pretransition and one posttransition. The first pretransition frequency category, Pretransition 1, consisted of $0.6-$ and $0.8-\mathrm{Hz}$ frequency plateaus. Pretransition 2 consisted of 1.0- and 1.2-Hz frequency plateaus; Pretransition 3 contained the plateaus just before those that contained the two modal transition frequencies. The posttransition category contained the average of the first two plateaus after the

\footnotetext{
${ }^{2}$ One may ask whether vision was indeed necessary for the phasing of limbs when the subjects' frequency of oscillation is paced by a metronome. By merely having the subjects start in an initial phase mode and entrain to the metronome pulse, if they performed the task perfectly, they should remain in the original phase mode. Following the same line of reasoning, one may ask whether the transition from one phase mode to another would occur without vision. Nonvisual control trials were performed for each subject pair. These showed a gradual shift in relative phase within a frequency of oscillation and no transitions of the kind noted for the alternate mode trials.
} 

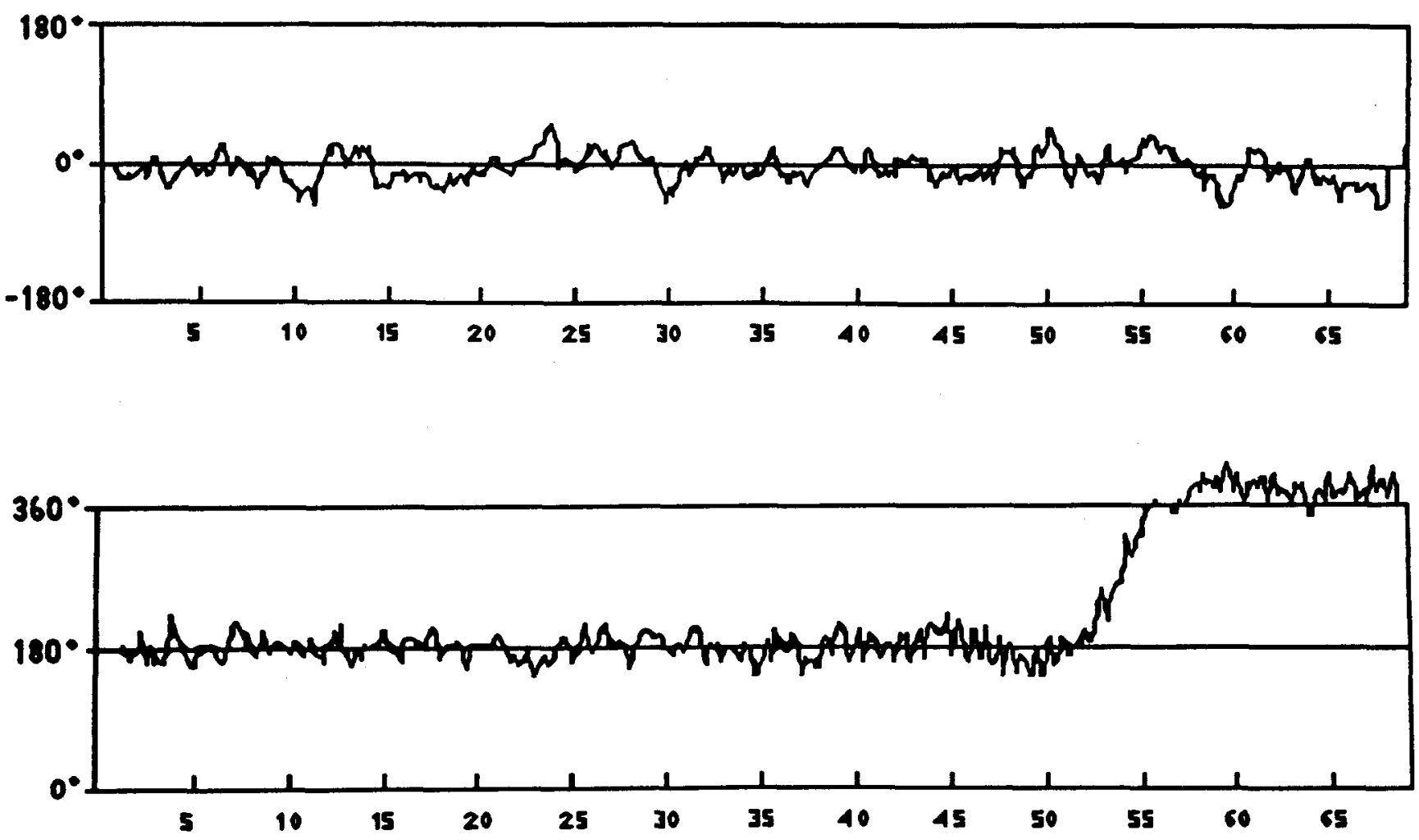

Figure 9. Examples of continuous relative phase time series for the symmetric (upper panel) and asymmetric (lower panel) conditions for Experiment 3. (The vertical axis is phase relation in degrees. The horizontal axis is time in seconds.)

transition. Because the frequency categories represent the ordinal position within a trial with respect to the transition, the variable representing these frequency categories will be called distance from transition. The mean standard deviations for each subject pair at each frequency plateau are provided in Table 1, along with comparable means calculated for the symmetric mode.

A three-way ANOVA with within-subject variables of phase mode (symmetric and alternate mode), frequency plateau length (5 s and $10 \mathrm{~s}$ ), and distance from transition (Pretransition 1-3 and posttransition) was performed on the standard deviation of relative phase. Figure 11 displays the means over the 3 subject pairs for the two frequency plateau conditions ( $5 \mathrm{~s}$ and $10 \mathrm{~s}$ ) combined. It is apparent that Pretransition 3 possesses a large increase in the standard deviation of relative phase for the alternate mode trials compared with the other alternate mode or symmetric mode means. The ANOVA revealed a significant Phase Mode $\times$ Distance From Transition interaction, $F(3,6)=10.127, M S_{\mathrm{e}}=14.902, p<.01$. Simple effects analyses found significant differences between phase modes at Pretransition 3, $F(1,2)=65.692, M S_{\mathrm{e}}=1.477, p<$ .02 , and the posttransition category, $F(1,2)=12.368, M S_{\mathrm{e}}=$ $.866, p<.05$, indicating that the phase fluctuations observed in the alternate mode were greater near the transition. Furthermore, a significant Fisher's protected $t$ test that compared the distances of Pretransition 2 and Pretransition $3(p<.01)$ demonstrates that the jump in standard deviation that is observed with the approach to the transition is statistically reliable. Another post hoc $t$ test also found the drop from Pretransition 3 to posttransition category to be significant ( $p$ $<.05$ ).

The fact that no three-way interaction of phase mode, distance from transition, and frequency plateau length was found is evidence that the observed increase in phase fluctuations occurs in both the 5-s and 10-s plateau conditions. To verify the latter, separate analyses of these two conditions were undertaken. Both of the 2 two-way ANOVAS with within variables of phase mode and distance from transition had significant two-way interactions (5-s plateaus: $F[3,6]=6.850$, $M S_{\mathrm{e}}=11.376, p<.05 ; 10$-s plateaus: $F[3,6]=5.108, M S_{\mathrm{e}}$ $=5.381, p<.05$ ). In each of these analyses, post hoc Fisher's protected $t$ tests revealed that the increase in standard deviation from the distance of Pretransition 2 to Pretransition 3 was significant ( $p<.01$, for both).

Critical fluctuations measured by power. The values of total power were averaged according to their distance from the transition, keeping separate pretransitional and posttransitional plateaus, and eliminating transitional plateaus. The means for each frequency plateau are provided in Table 2. A three-way ANOVA with within-subject variables of phase mode, frequency plateau length, and distance from transition was performed. A significant Phase Mode $\times$ Distance From Transition $\times$ Frequency Plateau Length interaction was found, $F(3,6)=12.673, M S_{\mathrm{e}}=.049, p<.01$, and is illustrated in 

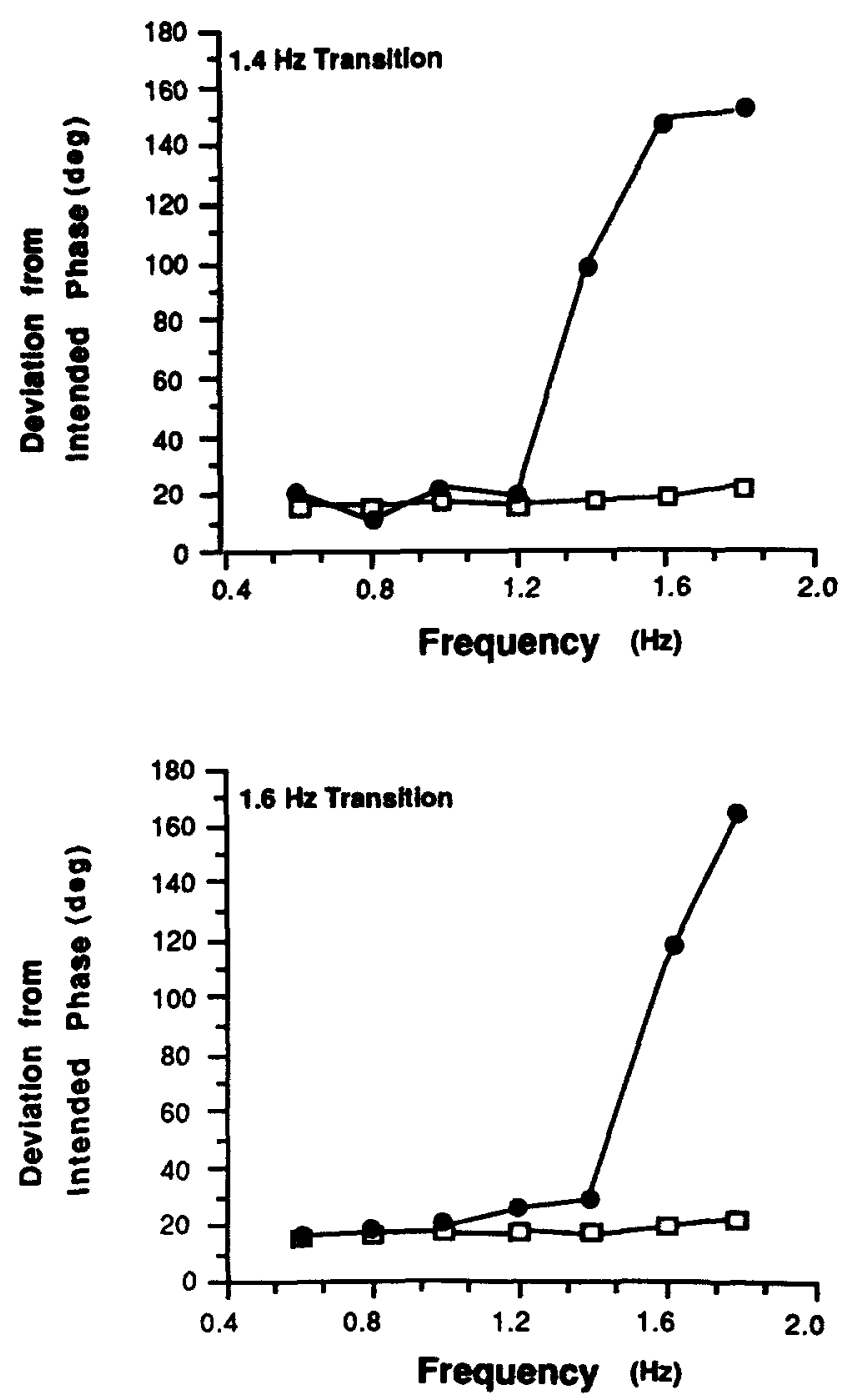

Figure 10. Mean deviations from intended relative phase as a function of frequency for the alternate mode trials in which the transitions occurred at the metronome frequency of $1.4 \mathrm{~Hz}$ (upper panel) and $1.6 \mathrm{~Hz}$ (lower panel). (Open squares are symmetric mode; closed circles are alternate mode.)

Figure 12. A simple effects analysis at each frequency plateau length revealed significant Phase Mode $\times$ Distance From Transition interactions, suggesting that the sudden increase in power at the last pretransition distance before the transition for the alternate mode trials occurs for both frequency plateau lengths: 5 -s plateaus, $F(3,6)=23.866, M S_{\mathrm{e}}=.073, p<.001$; and 10 -s plateaus, $F(3,6)=4.821, M S_{\mathrm{e}}=.054, p<.05$. The significant three-way interaction seems to have its origins in the behavior of the symmetric mode data at the two different frequency plateau lengths. Simple effects analyses indicate a significant rise in total power for the 5-s frequency plateau symmetric data across the distance categories, $F(3,6)=4.616$, $M S_{\mathrm{e}}=.148, p<.05$, but no change for the 10-s plateau symmetric mode data, $F(3,6)=0.341, M S_{\mathrm{e}}=.241, p>.05$. In contrast, the same analyses performed on the alternate mode data demonstrate a change in total power across the distance categories for both frequency plateau lengths. The change was significant for the 5-s plateaus, $F(3,6)=6.935$, $M S_{\mathrm{e}}=.231, p<.05$, but only marginally significant for the 10-s plateaus, $F(3,6)=3.961, M S_{\mathrm{e}}=.168, p=.07$.

Post hoc Fisher's protected $t$ tests found that the jump in total power from Pretransition 2 to Pretransition 3 was significant for both the 5- and 10-s plateau lengths $(p<.01$ for both) and the drop in power from Pretransition 3 to the posttransition distances was also significant for the 5 - and 10$s$ plateaus ( $p<.01$, for both). Also interesting is the degree of separation between the total power of the two phase modes at all distances from the transition, indicating that the alternate mode was in general more variable than the symmetric mode (Turvey et al., 1986). Simple effects analyses demonstrated that these differences were statistically significant at both 5 - and 10-s frequency plateau lengths (Table 3).

Hysteresis demonstration. Another characteristic feature of a physical bifurcation is that whether or not a bifurcation occurs and where in the range of control parameter values it occurs depends on the immediate history of the trajectory. The four hysteresis trials of SP 1 all had transitions from the alternate phase mode to the symmetric phase mode at the 1.6- $\mathrm{Hz}$ frequency plateau. The relative phase values of each frequency plateau were averaged across the trials and are displayed in Figure 13. As can be seen, decreasing the frequency after the transition did not lead to a switch back to the alternate mode of coordination. This was true for all four of the trials. The bifurcation was truly hysteretic.

\section{Discussion}

Experiment 3 has affirmed the preliminary results of Experiments 1 and 2 on visually coordinated rhythmic movements between two people: A breakdown in phase locking is seen in the initial alternate mode trials that leads to a transition from the relative phase values that are characteristic of the alternate phase mode to those characteristic of the symmetric phase mode as the frequency of oscillation increased. No such breakdown of phase locking is seen in the initial symmetric mode trials. These between-persons' results replicate the breakdown of phase locking found in within-person coordination (Baldissera, Cavallari, \& Civaschi, 1982; Cohen, 1971; Kelso, 1984; MacKenzie \& Patla, 1983). Following the reasoning that coordinated acts are produced by dynamically based functional synergies (Kugler, Kelso, \& Turvey, 1980, 1982), it has been suggested that such breakdowns correspond to an abrupt rearrangement of the topology of the dynamics underlying the coordination, that is, a physical bifurcation (Kelso \& Scholz, 1985).

Experiment 3 was undertaken to determine whether the between-persons transition from an alternate to a symmetric mode of coordination had the characteristic properties of a physical bifurcation. These properties are bimodality, inaccessibility, suddenness of jump, divergence, hysteresis, critical slowing down, and critical fluctuations. All of those that can be assessed with the phase transition methodology were found in the between-persons breakdown of phase locking. The lone exception, critical slowing down, requires a perturbation technique. It is uncertain how such a technique can be used given oscillating limb segments as massive as the lower legs. 
Table 1

Average Standard Deviation of Relative Phase for 5-s and 10-s Frequency Plateaus

\begin{tabular}{|c|c|c|c|c|c|c|c|c|}
\hline \multirow[b]{2}{*}{ Condition } & \multicolumn{8}{|c|}{ Frequency of oscillation } \\
\hline & 0.6 & 0.8 & 1.0 & 1.2 & 1.4 & 1.6 & 1.8 & 2.0 \\
\hline \multicolumn{9}{|c|}{ 5-s plateaus } \\
\hline \multicolumn{9}{|l|}{ Subject Pair 1} \\
\hline Alternate mode (pre) & 8.81 & 10.68 & 9.47 & 8.75 & 13.42 & 20.86 & 一 & 一 \\
\hline Alternate mode (post) & - & - & - & - & - & 一 & 14.27 & 18.03 \\
\hline Symmetric mode & 10.55 & 10.51 & 10.09 & 10.47 & 11.77 & 9.68 & 11.56 & 12.67 \\
\hline Subject Pair 2 & & & & & & & & \\
\hline Alternate mode (pre) & 10.27 & 12.43 & 13.13 & 13.55 & 16.13 & 17.25 & - & 一 \\
\hline Alternate mode (post) & - & - & - & - & - & 8.09 & 11.06 & - \\
\hline Symmetric mode & 8.16 & 9.60 & 9.29 & 8.70 & 8.63 & 9.72 & 7.34 & - \\
\hline \multicolumn{9}{|l|}{ Subject Pair 3} \\
\hline Alternate mode (pre) & 14.33 & 12.90 & 12.94 & 14.72 & 19.19 & 19.79 & 21.83 & - \\
\hline Alternate mode (post) & - & - & - & - & - & - & 12.79 & 15.24 \\
\hline Symmetric mode & 12.87 & 11.65 & 14.66 & 12.23 & 12.87 & 13.25 & 14.95 & 13.72 \\
\hline \multicolumn{9}{|c|}{ 10-s plateaus } \\
\hline \multicolumn{9}{|l|}{ Subject Pair 1} \\
\hline Alternate mode (pre) & 12.44 & 14.76 & 19.44 & 15.37 & - & - & - & - \\
\hline Alternate mode (post) & - & - & 13.42 & 14.59 & 13.17 & 16.75 & 20.56 & - \\
\hline Symmetric mode & 11.65 & 10.79 & 11.33 & 9.96 & 11.76 & 14.41 & 15.29 & - \\
\hline \multicolumn{9}{|l|}{ Subject Pair 2} \\
\hline Alternate mode (pre) & 12.93 & 10.67 & 11.01 & 13.77 & 12.69 & - & - & - \\
\hline Alternate mode (post) & - & - & - & - & 15.34 & 16.11 & 14.06 & - \\
\hline Symmetric mode & 12.73 & 12.36 & 11.90 & 12.05 & 12.31 & 13.55 & 13.58 & - \\
\hline \multicolumn{9}{|l|}{ Subject Pair 3} \\
\hline Alternate mode (pre) & 13.71 & 14.23 & 13.35 & 14.92 & 22.51 & 21.03 & - & - \\
\hline Alternate mode (post) & - & - & - & - & 16.00 & 19.99 & 17.20 & 一 \\
\hline Symmetric mode & 11.09 & 10.49 & 12.75 & 15.11 & 15.07 & 15.83 & 13.58 & - \\
\hline
\end{tabular}

Note. Values are measured in degrees.

As is the case for within-person coordination, there seem to be two stable modes of phasing limbs, either in phase (symmetric mode) or out of phase (alternate mode). The relative phase values in between these two modes (e.g., a relative phase of $90^{\circ}$ ) seem to be inaccessible in that they are exhibited only as transient states. Also, as with the withinperson case, this set of properties implicates the potential landscape of Haken et al. (1985) presented in Figure 14 that

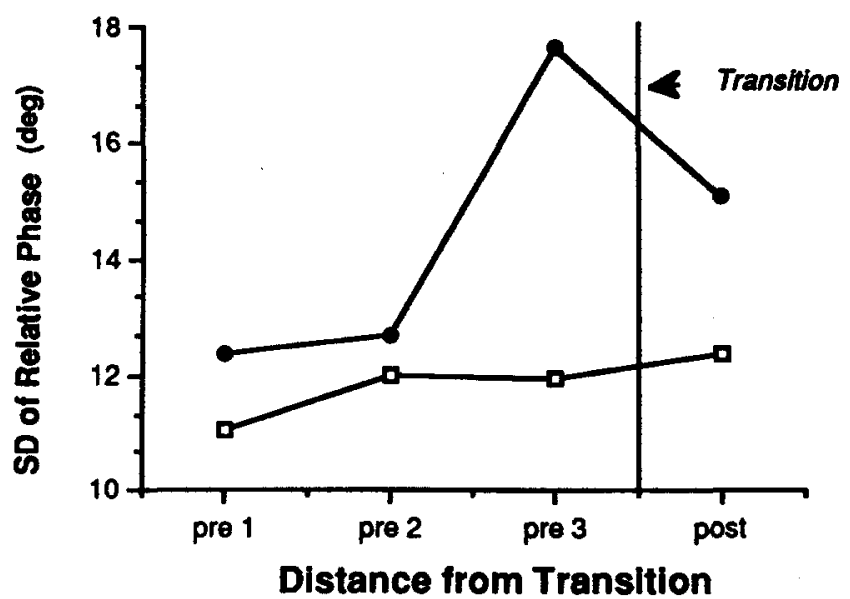

Figure 11. Standard deviation of relative phase as a function of phase mode and distance from transition. (Data are the 5-s and 10-s plateaus combined. Open squares are symmetric mode; closed circles are alternate mode.) has two minima with a sharp gradient between them; it is provided by the function $V(\Phi)=-a \cos (\Phi)-b \cos (2 \Phi)$, where $\mathrm{d} \Phi / \mathrm{d} t=\partial V / \partial \Phi$. One minimum of the landscape represents the steady state symmetric phasing at $0^{\circ}$ relative phase $(\Phi)$, the other represents the steady state alternate phasing at $180^{\circ}$, and the gradient represents the inaccessible, transient regions of relative phase. More formally, the minima are where the rate of change of the potential and, hence, of relative phase, is zero $(\partial V / \partial t=0)$. The steep slope is where the rate of change of the potential and of relative phase is much greater than zero $(\partial V / \partial t \gg 0)$.

Past research has demonstrated that the two phase modes are differentially stable: The symmetric mode is in general more stable than the alternate mode (Kelso \& Scholz, 1985; Scholz, 1986; Turvey et al., 1986). Our studies suggest that the same is true for movements coordinated between two people. Not only does the alternate mode phase locking break down at higher frequencies but the variability of relative phase in the alternate mode trials in Experiment 3 is greater overall than in the symmetric mode trials (Figures 11 and 12), although significance was found only in the total power measure (Table 3).

A sudden jump from the alternate mode to the symmetric mode was seen for all initial alternate mode trials as the frequency of oscillation was scaled in Experiments 2 and 3. This jump is a divergent response of relative phase to the scaling of the control parameter, namely, frequency of oscillation: A small change in the frequency of oscillation that previously led to a small change in relative phase leads to a 
Table 2

Average Total Power of Relative Phase for 5-s and 10-s Frequency Plateaus

\begin{tabular}{|c|c|c|c|c|c|c|c|c|}
\hline \multirow[b]{2}{*}{ Condition } & \multicolumn{8}{|c|}{ Frequency of oscillation } \\
\hline & 0.6 & 0.8 & 1.0 & 1.2 & 1.4 & 1.6 & 1.8 & 2.0 \\
\hline \multicolumn{9}{|c|}{ 5-s plateaus } \\
\hline \multicolumn{9}{|l|}{ Subject Pair 1} \\
\hline Alternate mode (pre) & - & 5.04 & 5.11 & 5.02 & 5.66 & 6.72 & - & - \\
\hline Alternate mode (post) & - & - & - & - & - & 4.97 & 4.81 & - \\
\hline Symmetric mode & - & 2.79 & 2.63 & 2.84 & 3.00 & 3.37 & 3.69 & 4.69 \\
\hline \multicolumn{9}{|l|}{ Subject Pair 2} \\
\hline Alternate mode (pre) & - & 5.81 & 5.40 & 5.26 & 6.07 & 6.96 & - & \\
\hline Alternate mode (post) & - & - & - & - & - & 2.49 & 4.42 & \\
\hline Symmetric mode & - & 2.93 & 2.00 & 3.05 & 2.63 & 2.20 & 3.64 & \\
\hline \multicolumn{9}{|l|}{ Subject Pair 3} \\
\hline Alternate mode (pre) & 一 & 5.12 & 4.97 & 5.66 & 5.98 & 6.48 & 7.24 & - \\
\hline Alternate mode (post) & 一 & - & - & - & - & - & 3.99 & 5.68 \\
\hline Symmetric mode & - & 2.96 & 3.82 & 3.60 & 4.05 & 3.46 & 4.84 & 3.61 \\
\hline \multicolumn{9}{|c|}{ 10-s plateaus } \\
\hline \multicolumn{9}{|l|}{ Subject Pair 1} \\
\hline Alternate mode (pre) & 3.92 & 4.03 & 4.74 & 5.40 & - & - & - & - \\
\hline Alternate mode (post) & - & - & 4.46 & 3.69 & 3.28 & 4.01 & 5.07 & - \\
\hline Symmetric mode & 2.73 & 2.72 & 2.27 & 3.05 & 3.05 & 3.81 & 3.89 & - \\
\hline \multicolumn{9}{|l|}{ Subject Pair 2} \\
\hline Alternate mode (pre) & 5.91 & 4.53 & 4.78 & 4.98 & 5.73 & - & - & - \\
\hline Alternate mode (post) & - & - & - & - & 3.06 & 3.56 & 3.28 & - \\
\hline Symmetric mode & 2.82 & 3.01 & 2.95 & 2.84 & 2.87 & 3.83 & 3.04 & - \\
\hline \multicolumn{9}{|l|}{ Subject Pair 3} \\
\hline Alternate mode (pre) & 4.43 & 5.13 & 4.82 & 4.91 & 5.90 & 6.50 & - & - \\
\hline Alternate mode (post) & - & - & - & - & 5.19 & 6.04 & 5.21 & - \\
\hline Symmetric mode & 2.69 & 2.58 & 2.96 & 3.95 & 3.75 & 4.26 & 3.68 & - \\
\hline
\end{tabular}

Note. Values are measured in $\log _{e}\left(\mathrm{deg}^{2}\right)$.

very large change in relative phase at the point of divergence. In a dynamical system, a sudden jump is brought about by virtue of the control parameter's changing of the potential landscape, making previously stable states unstable. Figure 14 shows how a critical ratio of the parameters $a$ and $b$ produces the final annihilation of the steady state potential minimum at $180^{\circ}$ and $\partial V / \partial t \gg 0$. The gradual evolution of the original landscape's topology then produces a sudden and divergent change in the phasing of the limbs. Some variability in the frequency at which the transition occurred was found. This variability is also found in within-person bifurcation. Scholz (1986) documents both inter- and intrasubject variability of the transition frequency. These findings do not necessarily undermine the lawfulness of the system producing the bifurcation. Indeed, a stochastic element is an integral part of the modeling of all bifurcation phenomena (Schöner et al., 1986). The variability of transition frequency, however, does pose methodological problems in that certain trials must be analyzed separately from others or trials must be grouped according to some unifying factor, as was done in our experiments.

Generally, reversing the control parameter scaling after a bifurcation produces a jump back to the original mode of functioning. If this return jump occurs at a different control parameter value or if it does not occur at all, the bifurcation is hysteretic. After the transition to the symmetric mode occurred in the initial alternate mode trials, decreasing the frequency of oscillation did not lead to a transition back to the alternate mode of coordination. This form of hysteresis was also found with the within-person bifurcation (Kelso \&
Kay, 1987) and follows explicitly from the Haken et al. (1985) model and computer simulations.

An increase in fluctuations before the transition is another property of physical bifurcations. For both measures of variability - standard deviation and total power of phase-Experiment 3 found a significant increase in relative phase fluctuation at the frequency plateaus near the transition. Furthermore, critical fluctuations were found for both 5- and 10-s plateaus. How does a physical account of the transition predict these fluctuations? As the transition to another mode of functioning is approached, the attractor underlying the present mode becomes less and less stable. Returning to Figure 14 , as the transition is approached the potential well at $180^{\circ}$ becomes flatter and flatter. Assuming that the system is subjected to stochastic perturbations, as is reasonable for complex systems (Haken, 1978), a perturbation of a given magnitude will cause a greater deviation in the flatter potential well near the transition than in the steeper sided potential well away from the transition. Hence, one could expect to see an increase in fluctuations as the transition is approached if the jump has a dynamical basis.

Investigation of within-person bimanual finger oscillations found critical fluctuations for 5-s but not 10-s frequency plateaus (Kelso \& Scholz, 1985; Scholz, 1986). This apparent lack of prediction has a ready explanation (Scholz et al., 1987) in the difference in the conventions (see introduction to Experiment 3) that are operating in the situations of the two studies. From the relations among time scales (Schöner et al., 1986), it is evident that what dictates whether one convention 

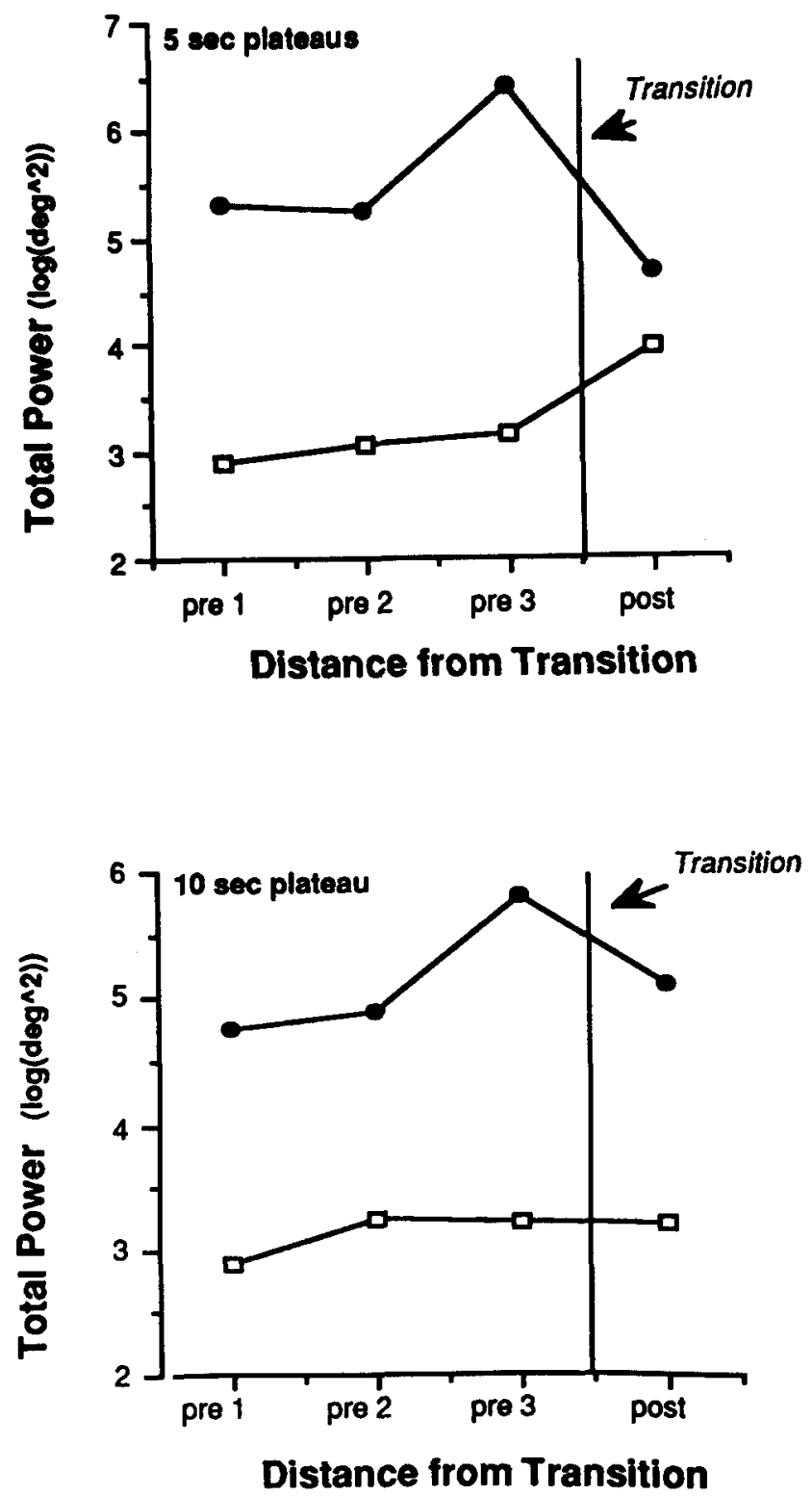

Figure 12. Total power of relative phase as a function of phase mode and distance from the transition frequency. (Open squares are symmetric mode; closed circles are alternate mode.)

or another will hold is the magnitude of the first passage time relative to the control parameter change time (in this study, it is the frequency plateau time of 5 or $10 \mathrm{~s}$ ). The first passage time is the time it takes to move the system probabilistically from one stable state to another (i.e., without criticality). If the first passage time is greater than the control parameter change time, then the delay convention will hold and critical fluctuations will be observed. If the first passage time is less than the control parameter change time, then the Maxwell convention will hold, the transition will occur before the first mode has become unstable, and critical fluctuations will not be observed. Scholz et al. (1987) estimated that the first passage time for their 10-s frequency plateau experiment was less than the 10-s control parameter change time, but the first passage time for the 4-s plateau experiment was much more than the 4-s control parameter change time. In short, the two experiments discussed by Scholz et al. straddle the two bifurcation conventions. In Experiment 3, the control parameter change time (the frequency plateau duration) was manipulated in order to potentially accommodate the two conventions. Because critical fluctuations were observed at both the 5-s and 10-s frequency plateaus lengths, however, the delay convention appears to have been operating in each.

The between-persons and within-person transition results differ with respect to the observed fluctuations. This is perhaps not as dichotomous as it may appear. Although critical fluctuations were found in both frequency plateau length conditions, there is some indication that the degree of criticality (namely, degree of instability) exhibited was different in the two conditions and that less criticality was observed in the 10-s frequency plateau trials. Scholz et al. (1987) provided a metric for indexing criticality,

$$
\begin{aligned}
& \text { criticality index }=S D_{\text {alternate }} / S D_{\text {symmetric }} \\
& =\sqrt{ } \tau_{\text {relaxation alternate }} / \tau_{\text {relaxation symmetric, }}
\end{aligned}
$$

where $S D$ is the standard deviation, $\tau_{\text {relaxation alternate }}$ is the relaxation time of the plateau just prior to the transition for alternate mode, and $\tau_{\text {relaxation symmetric }}$ is the relaxation time of all plateaus for the symmetric mode. In one experiment in which critical fluctuations (and, hence, a great amount of criticality) were observed (Kelso \& Scholz, 1985), the ratio of the standard deviations equaled 2.13; in another experiment in which critical fluctuations were not observed, the ratio of relaxation times was approximately 1.5 (Scholz, 1986). In the between-persons coordination observed in Experiment 3, the ratios of standard deviations exhibited by SP 1, SP 2, and SP 3 were, respectively, $1.908,1.925$, and 1.625 for the 5 -s plateaus and $1.597,1.089$, and 1.678 for the 10 -s plateaus (using the plateau with the greatest critical fluctuations). The 10-s plateau ratios were lower than the 5-s plateau overall, indicating that the average criticality achieved in the longer plateaus was less than in the shorter plateaus (although only one of these values is less than 1.5). Additional evidence for this fact is that the time taken for the transition to occur is longer for the 10 -s plateau trials than for the 5 -s plateau trials.

Table 3

"Phase Mode at Distance From Transition" Simple Effects Tests for Total Power

\begin{tabular}{cccc}
\hline & \multicolumn{3}{c}{ Results } \\
\cline { 2 - 4 } Effect & $d f$ & $M S_{e}$ & $F$ \\
\hline 5-s plateaus & & & \\
Pretransition 1 & 1,2 & 0.078 & $113.29^{* *}$ \\
Pretransition 2 & 1,2 & 0.133 & $55.80^{*}$ \\
Pretransition 3 & 1,2 & 0.373 & $42.54^{*}$ \\
Posttransition & 1,2 & 0.003 & $204.81^{* *}$ \\
10-s plateaus & & & \\
Pretransition 1 & 1,2 & 0.134 & $38.36^{*}$ \\
Pretransition 2 & 1,2 & 0.057 & $69.08^{*}$ \\
Pretransition 3 & 1,2 & 0.123 & $81.16^{*}$ \\
Posttransition & 1,2 & 0.187 & $28.62^{*}$ \\
\hline
\end{tabular}

${ }^{*} p<.05$. $^{* *} p<.01$. 


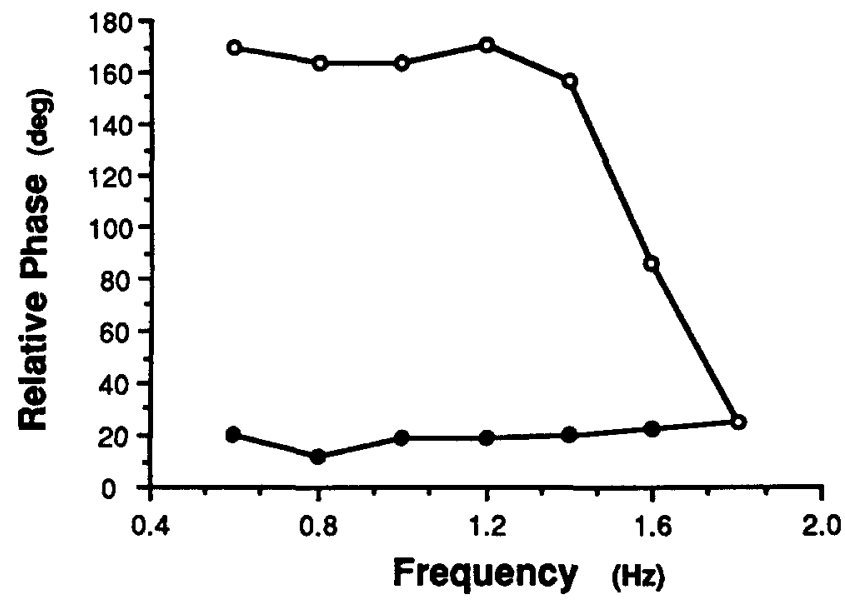

Figure 13. Relative phase of each frequency plateau for hysteresis trials. (Open circles are for increasing frequency; closed circles are for decreasing frequency.)

Of some concern is the fairly small increase in the standard deviation of the relative phase in the between-persons bifurcation compared with the increase found in the within-person bifurcation. The fluctuation increase between the second to last and the last pretransition frequency plateau was approximately $15^{\circ}$ for the Kelso and Scholz (1985) 4-s plateau data. For Experiment 3 , the increase was approximately $4^{\circ}$. This difference can be attributed somewhat to the difference in baseline variability of the alternate phase mode in the between-persons and within-person experiments. The baseline variability was approximately $11^{\circ}$ for Experiment 3 and approximately $7^{\circ}$ for Kelso and Scholz. But the difference is mostly a result of the depression of values in Pretransition 3 because, in Experiment 3, these values are means across subjects and means of the two plateaus preceding the modal transition frequency plateaus. The fluctuations in the first of these two pretransition plateaus are not always critical, because the transition was not to occur in the next plateau but rather the one after that. As such, the distance from transition categorization of the frequency plateaus as an independent variable results in a more conservative test of critical fluctuations.

Also of some concern is the role that intention played in the transitional behavior between the two phase modes. The question can be asked whether the subjects purposefully switched their mode of phasing from the alternate mode to the symmetric mode. For example, they could have been biased by the instructions and previous knowledge that some transition would occur and, as a consequence, induce a transition. Two points should be mentioned in this regard. First, it does not seem that intention can totally supersede the dynamical constraints that produce the transition between the phase modes. A recent study of within-person limb phasing (Kelso, Scholz, \& Schöner, 1988; Schöner \& Kelso, 1988b) investigated the intentional switching between the two phase modes and found that the time taken for the intentional transition to occur was greater for the transition from symmetric mode to alternate mode than for the transition from alternate mode to symmetric mode. Kelso et al. (1988) interpreted this result as the intentional transition having to op- erate within the constraints of the intrinsic dynamics underlying the phasing of limbs.

Second, intentional switching from the alternate mode to symmetric phase mode occurs much faster $(0.41 \mathrm{~s}$; Kelso et al., 1988) than does the nonintentional within-person switching (4.28 s; Scholz, 1986) or the between-persons switching ( $3.34 \mathrm{~s}$; Experiment 3). Assuming that the ability to intentionally control the phasing of limbs is about equal within a person and between two people, the switching times observed indicate that our study's subject pairs were not intentionally producing a transition from the alternate to the symmetric mode of phasing.

Furthermore, a cognitive theorist may choose to explain the apparent differential stability of the two phase modes in terms of processing load: Maintaining the alternate mode requires more cognitive control or attention than maintaining the symmetric mode. Following this line of argument, however, Experiment 3 would have produced a greater processing load than Experiment 2, because the subjects not only were coordinating the phasing of their limbs but also were tracking the metronome. One would expect that this increase in processing load would have caused a breakdown of the alternate mode at a lower frequency of oscillation in the experiment with the greater processing load. But the opposite result occurred: The mean transition frequency was $1.301 \mathrm{~Hz}$ for Experiment 2 and 1.529 for Experiment 3.

\section{Conclusions}

This study has attempted to assess whether the phasing of limbs between two people can be characterized by the same properties found in the phasing of limbs within a person. It can be concluded that the same principles underlie both situations. The properties of interest from the within-person coordination include the following. Two distinct modes of phasing limbs are observed, namely, the alternate and symmetric modes. The alternate mode is less stable than the symmetric mode, as indicated by a breakdown of phasing to the symmetric mode at high frequencies of oscillation. At some critical frequency, the alternate mode phasing can no longer be maintained and only the symmetric mode phasing can be stably coordinated.

A similar set of properties was found for the betweenpersons phasing of limbs. In Experiment 1, when subjects were instructed to remain in the initial mode of phasing, we found that the alternate mode in the between-persons phasing of limbs was less stable than the symmetric mode as the frequency of oscillation increased. Furthermore, the instability observed at the highest frequencies represented a breakdown of the alternate phase mode. There was evidence from the number of nonsteady state cycles that the alternate mode was exhibiting a breakdown in phasing that was indicative of a transitionlike behavior; that is, subject pairs often "visited" the symmetric mode during the breakdown. In Experiment 2 , when the subject pair was instructed to remain in the symmetric mode after a breakdown in the alternate mode phasing, a transition from the alternate mode to the symmetric mode of phasing occurred regularly as it does in withinperson bimanual coordination. Experiment 3 then tested this between-persons phase transition to see if it had the characteristic properties of a physical bifurcation. The criterial prop- 

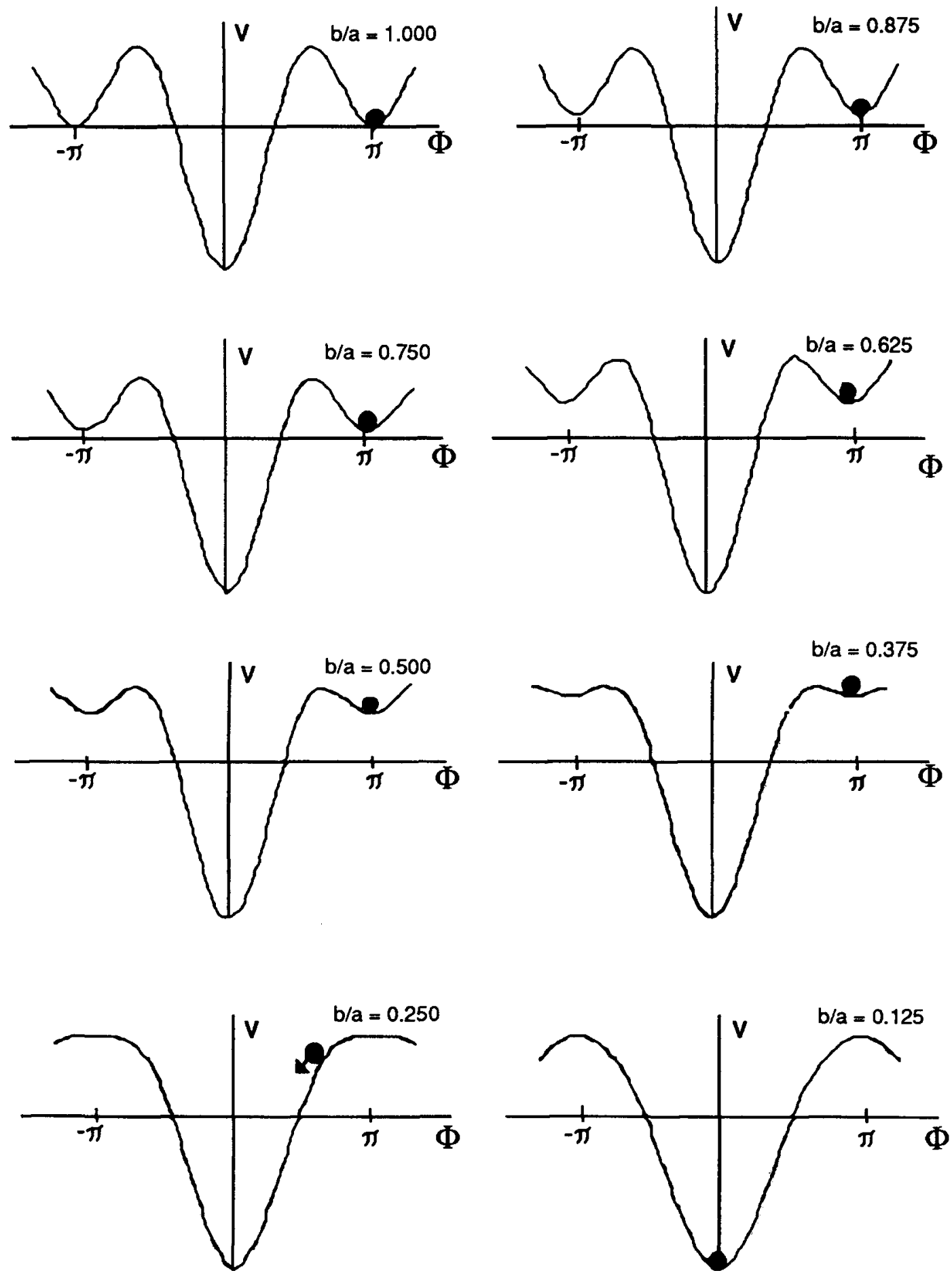

Figure 14. Proposed potential function underlying the spontaneous transition from alternate phase mode to symmetric phase mode. (Adapted from Haken, Kelso, \& Bunz, 1985.)

erties of bimodality, inaccessibility, suddenness of jump, divergence, hysteresis, and critical fluctuations were all observed. Hence, it can be concluded that the same principles underlie within-person and between-persons phase transitions.

The first point to be made from this investigation is that the breakdown in phase locking observed for the alternate mode phasing of limbs between two people succumbs to a dynamical characterization. A dynamically based functional synergy is formed across the two individuals. Coordination between two individuals arises through the mutual harnessing of the attractor dynamics underlying the relative phasing of the limbs. Furthermore, the evolution of this control structure is a consequence of the topology of the potential underlying 
it. Hence, a principled basis for the spontaneous change in the coordination of two people is provided.

Although this article comprises laboratory studies of a novel coordination of limbs, the possible ramifications it has for human interaction in general are quite profound. They follow from considering the experimental results from the perspective of the ecological theory of perception and action (Gibson, 1979; Turvey \& Carello, 1986; Turvey \& Kugler, 1984; Turvey, Shaw, Reed, \& Mace, 1981), which attempts to provide a law-based account of animal-environment interactions. One ramification is that the interaction of an animal with its environment or, equally, the interaction of one individual with another, is not created solely from the cognitive and/or neural structures of the individual but arises in a context of constraints. Constraints here mean simply the way that things can fit together physically, the patterns of nature (Stevens, 1974). Human interactions involving the mutual timings and phasings of behaviors must follow these constraints (Beek, 1989). Consequently, cooperative states that arise in interpersonal settings ought to be conceptualized as complex physical systems conforming to the same modes of organization as nature generally. This conceptualization does not rule out new phenomena at the social scale of interaction. Rather, it is an assertion that the structuring of these phenomena is in all likelihood due to very general principles that function across scales.

The second major point of this study is that dynamical control structures can be formed over informational structures. When optical information about the other person's leg is eliminated, neither phase locking nor, relatedly, the bifurcation from the alternate to symmetric mode can be observed. The establishment, maintenance, and dissolution of a between-persons coordinative state rests on information about the coordinative state, its kinematics, and its dynamics. Indeed, the attractor layout depicted in Figure 14 is essentially dependent on information for its formation and evolution. More specifically, coordinative interactions between two individuals occur in terms of observables that are best described as informational observables (Kugler \& Turvey, 1987; Turvey, in press). These observables are expressed as kinematic patternings of a low-energy field, namely, the optic array, specific to properties of the moving limbs (Kugler \& Turvey, 1987; Kugler, Turvey, Carello, \& Shaw, 1985).

Because informational interactions are the foundation of perceiving-acting cycles defined over an animal-environment system, a complete theory of informational interactions (Schöner \& Kelso, 1988a; Shaw \& Kinsella-Shaw, 1988) is perhaps the greatest theoretical challenge to the dynamically based account of behavior envisioned by the ecological approach to perception and action (Turvey, 1988). The present study provides evidence for the operation of dynamical systems in at least one situation in which a material (i.e., kinetic) linkage of components is absent and an informational linkage is the only explanation of the resultant order.

\section{References}

Asch, S. E. (1952). Social psychology. Englewood Cliffs, NJ: PrenticeHall.

Baldissera, F., Cavallari, P., \& Civaschi, P. (1982). Preferential cou- pling between voluntary movements of ipsilateral limbs. Neuroscience Letters, 34, 95-100.

Beek, P. J. (1989). Timing and phase locking in cascade juggling. Ecological Psychology, 1, 55-96.

Beek, P. J., \& Beek, W. J. (1988). Tools for constructing dynamical models of rhythmic movement. Human Movement Science, 7 , 301-342.

Bernieri, F., Reznick, J. S., \& Rosenthal, R. (1988). Synchrony, pseudosynchrony, and dissynchrony: Measuring the entrainment process in mother-infant interactions. Journal of Personality and Social Psychology, 54, 243-253.

Bingham, G. P. (1987). Kinematic form and scaling: Further investigations on the visual perception of lifted weights. Journal of Experimental Psychology: Human Perception and Performance, 13. 155-177.

Bramble, D. M., \& Carrier, D. R. (1983). Running and breathing in mammals. Science, 219, 251-256.

Cohen, L. (1971). Synchronous bimanual movements performed by homologous and non-homologous muscles. Perceptual and Motor Skills, 32, 639-644.

Davis, M. (Ed.). (1982). Interaction rhythms: Periodicity in communicative behavior. New York: Human Science Press.

Fitch, H. L., Tuller, B., \& Turvey, M. T. (1982). The Bernstein perspective: 3 . Tuning of coordinative structures with special reference to perception. In J. A. S. Kelso (Ed.), Human motor behavior: An introduction (pp. 271-287). Hillsdale, NJ: Erlbaum.

Gibson, J. J. (1966). The senses considered as perceptual systems. Boston: Houghton Mifflin.

Gibson, J. J. (1979). The ecological approach to visual perception. Boston: Houghton Mifflin.

Gilmore, R. (1979). Catastrophe time scales and conventions. Physical Review $A, 20,2510-2515$.

Gilmore, R. (1981). Catastrophe theory for scientists and engineers. New York: Wiley.

Haken, H. (1978). Synergetics: An introduction (2nd ed.). Heidelberg, Federal Republic of Germany: Springer-Verlag.

Haken, H. (1983). Advanced synergetics. Heidelberg, Federal Republic of Germany: Springer-Verlag.

Haken, H., Kelso, J. A. S., \& Bunz, H. (1985). A theoretical model of phase transitions in human hand movements. Biological Cybernetics, 51, 347-356.

Hoyt, D. F., \& Taylor, C. R. (1981). Gait and the energetics of locomotion in horses. Nature, 292, 239-240.

Kay, B. A. (1986). Dynamic modelling of rhythmic limb movements: Converging on a descriptor of the component oscillator. Unpublished doctoral dissertation, University of Connecticut, Storrs.

Kay, B. A. (1988). The dimensionality of movement trajectories and the degrees of freedom problem: A tutorial. Human Movement Science, 7, 343-364.

Kay, B. A., Kelso, J. A. S., Saltzman, E. S., \& Schöner, G. (1987). The space-time behavior of single and bimanual rhythmical movements. Journal of Experimental Psychology: Human Perception and Performance, 13, 564-583.

Kelso, J. A. S. (1984). Phase transitions and critical behavior in human bimanual coordination. American Journal of Physiology: Regulatory, Integrative and Comparative, 246, R 1000-R 1004.

Kelso, J. A. S., Holt, K. G., Rubin, P., \& Kugler, P. N. (1981). Patterns of human interlimb coordination emerge from the properties of nonlinear limit cycle processes: Theory and data. Journal of Motor Behavior, 13, 226-261.

Kelso, J. A. S., \& Kay, B. A. (1987). Information and control: A macroscopic analysis of perception-action coupling. In $\mathbf{H}$. Heuer \& A. F. Sanders (Eds.), Tutorials in perception and action (pp. 332). Amsterdam: North-Holland.

Kelso, J. A. S., \& Scholz, J. P. (1985). Cooperative phenomena in biological motion. In H. Haken (Ed.), Complex systems: Opera- 
tional approaches in neurobiology, physical systems and computers (pp. 124-149). Berlin, Federal Republic of Germany: SpringerVerlag.

Kelso, J. A. S., Scholz, J. P., \& Schöner, G. (1986). Nonequilibrium phase transitions in coordinated biological motion: Critical fluctuations. Physics Letters, 118, 279-284.

Kelso, J. A. S., Scholz, J. P., \& Schöner, G. (1988). Dynamics governs switching among patterns of coordination in biological movement. Physics Letters, 134, 8-12.

Kelso, J. A. S., Schöner, G., Scholz, J. P., \& Haken, H. (1987). Phaselocked modes, phase transitions and component oscillators in biological motion. Physica Scripta, 25, 79-87.

Kendon, A. (1970). Movement coordination in social interaction: Some examples. Acta Psychologica, 32, 1-25.

Kugler, P. N. (1986). A morphological perspective on the origin and evolution of movement patterns. In M. G. Wade \& H. T. A. Whiting (Eds.), Motor development in children: Aspects of coordination and control (pp. 459-525). The Hague: Nijhoff.

Kugler, P. N., Kelso, J. A. S., \& Turvey, M. T. (1980). On the concept of coordinative structures as dissipative structures: 1 . Theoretical lines of convergence. In G. E. Stelmach \& J. Requin (Eds.), Tutorials in motor behavior (pp. 3-47). Amsterdam: North-Holland.

Kugler, P. N., Kelso, J. A. S., \& Turvey, M. T. (1982). On the control and coordination of naturally developing systems. In J. A. S. Kelso \& J. E. Clark (Eds.), The development of movement control and coordination (pp. 5-78). New York: Wiley.

Kugler, P. N., \& Turvey, M. T. (1987). Information, natural law and the self-assembly of rhythmic movement. Hillsdale, NJ: Erlbaum.

Kugler, P. N., \& Turvey, M. T. (1988). Self-organization, flow fields, and information. Human Movement Science, 7, 97-130.

Kugler, P. N., Turvey, M. T., Carello, C., \& Shaw, R. (1985). The physics of controlled collisions: A reverie about locomotion. In W. H. Warren \& R. E. Shaw (Eds.), Persistence and change (pp. 195229). Hillsdale, NJ: Erlbaum.

Kugler, P. N., Turvey, M. T., \& Shaw, R. E. (1982). Is the "cognitive impenetrability" criterion invalidated by contemporary physics? Behavioral and Brain Sciences, 5, 303-306.

MacKenzie, C. L., \& Patla, A. E. (1983). Breakdown in rapid bimanual finger tapping as a function of orientation and phasing. Society for Neurosience. (Abstract).

Newtson, D., Hairfield, J., Bloomingdale, J., \& Cutino, S. (1987). The structure of action and interaction. Social Cognition, 5, 191237.

Press, W. H., Flannery, B. P., Teukolsky, S. A., \& Vetterling, W. T. (1988). Numerical recipes in $C$ : The art of scientific computing. Cambridge, England: Cambridge University Press.

Prigogine, I. (1980). From being to becoming: Time and complexity in the physical sciences. San Francisco: Freeman.

Prigogine, 1., \& Stengers, I. (1984). Order out of chaos: Man's new dialogue with nature. New York: Bantam.

Rosenblum, L. D., \& Turvey, M. T. (1988). Maintenance tendency in coordinated thythmic movements: Relative fluctuation and phase. Neuroscience, 27, 289-300.

Runeson, S., \& Frykholm, G. (1981). Visual perception of lifted weight. Journal of Experimental Psychology: Human Perception and Performance, 7, 733-740.

Saltzman, E. L. (1987). Task dynamic coordination of the speech articulators: A preliminary model. Experimental Brain Research, $15,129-144$.

Saltzman, E. L., \& Kelso, J. A. S. (1987). Skilled actions: A taskdynamic approach. Psychological Review, 94, 84-106.

Scholz, J. P. (1986). A nonequilibrium phase transition in human bimanual movement: Test of a dynamical model. Unpublished doctoral dissertation, University of Connecticut, Storrs.

Scholz, J. P., Kelso, J. A. S., \& Schöner, G. (1987). Nonequilibrium phase transitions in coordinated biological motion: Critical slowing down and switching time. Physics Letters, 123, 390-394.

Schöner, G., Haken, H., \& Kelso, J. A. S. (1986). A stochasic theory of phase transitions in human hand movement. Biological Cybernetics, 53, 247-257.

Schöner, G., \& Kelso, J. A. S. (1988a). Dynamic pattern generation in behavioral and neural systems. Science, 239, 1513-1520.

Schöner, G., \& Kelso, J. A. S. (1988b). A dynamic pattern theory of behavioral change. Journal of Theoretical Biology, 135, 501-524.

Schöner, G., \& Kelso, J. A. S. (1988c). Dynamic patterns of biological coordination: Theoretical strategy and new results. In J. A. S. Kelso, A. J. Mandell, \& M. F. Shlesinger (Eds.), Dynamic patterns in complex systems (pp. 77-102). Singapore: World Scientific.

Shaw, R. E., \& Kinsella-Shaw, J. (1988). Ecological mechanics: A physical geometry for intentional constraints. Human Movement Science, 7, 155-200.

Stafford, F. S., \& Barnwell, G. M. (1985). Mathematical models of central pattern generators in locomotion: I. Current problems. Journal of Motor Behavior, 17, 3-26.

Stevens, P. S. (1974). Patterns of nature. Boston: Little, Brown.

Thompson, J. M. T., \& Stewart, H. B. (1986). Nonlinear dynamics and chaos. New York: Wiley.

Todd, J. T., \& Warren, W. H. (1982). Visual perception of relative mass in dynamic events. Perception, 11, 325-335.

Turvey, M. T. (1988). Simplicity from complexity: Archetypal regimes and smart perceptual mechanisms as execution-driven phenomena. In J. A. S. Kelso, A. J. Mandell, \& M. F. Shlesinger (Eds.), Dynamic patterns in complex systems (pp. 327-347). Singapore: World Scientific.

Turvey, M. T. (in press). The challenge of a physical account of action: A personal view. In H. T. A. Whiting, O. G. Meijer, \& P. C. W. van Wieringen (Eds.), A natural-physical approach to movement. Amsterdam: Free University Press.

Turvey, M. T., \& Carello, C. (1986). The ecological approach to perceiving-acting: A pictorial essay. Acta Psychologica, 63, 133155.

Turvey, M. T., \& Kugler, P. N. (1984). An ecological approach to perception and action. In H. T. A. Whiting (Ed.), Human motor actions: Bernstein reassessed (pp. 373-410). Amsterdam: NorthHolland.

Turvey, M. T., Rosenblum, L. D., Schmidt, R. C., \& Kugler, P. N. (1986). Fluctuations and phase symmetry in coordinated rhythmic movements. Journal of Experimental Psychology: Human Perception and Performance, 12, 564-583.

Turvey, M. T., Shaw, R. E., Reed, E. S., \& Mace, W. M. (1981). Ecological laws of perceiving and acting: In reply to Fodor and Pylyshyn (1981). Cognition, 9, 237-304.

von Holst, E. (1973). Relative coordination as a phenomenon and as a method of analysis of central nervous function. In R. Martin (Ed. and Trans.), The collected papers of Erich von Holst: Vol 1. The behavioral physiology of animal and man. Coral Gables, FL: University of Miami Press. (Original work published in 1939)

Warren, W. H. (1984). Perceiving affordances: Visual guidance of stair climbing. Journal of Experimental Psychology: Human Perception and Performance, 10, 683-703.

Yaminishi, J., Kawato, M., \& Suzuki, R. (1979). Studies on human finger tapping neural networks by phase transition curves. Biological Cybernetics, 33, 199-208.

Yaminishi, J., Kawato, M., \& Suzuki, R. (1980). Two coupled oscillators as a model for the coordinated finger tapping by both hands. Biological Cybernetics, 37, 219-225.

Received October 4, 1988

Revision received July 17, 1989

Accepted July 18, 1989 